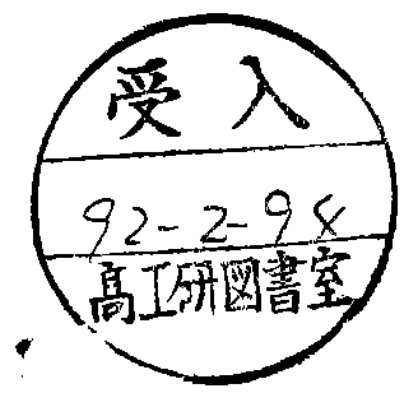

\title{
Development and Test of a Large Silicon Strip System for a Hadron Collider Beauty Trigger
}

\author{
(The P238 Collaboration)
}

J. Ellett, S. Erhan, P. Kreuzer ${ }^{1}$, D. Lynn, M. Medinnis, P. Schlein, J. Zweizig

University of California*), Los Angeles, U.S.A.

A. Czermak ${ }^{2}$, G. Engelmann, O. Klingsheim, K. Ley,

M. Price, A. Rudge, Hartmut Wahl, P. Weilhammer

CERN, Geneva, Switzerland

$$
\text { G. Borreani }{ }^{3}
$$

University of Ferrara and INFN, Italy

W. Hofmann, B. Wilkens

Max Planck Institut für Kernphysik, Heidelberg, Germany

M. Calvetti, M. Punturo

University of Perugia and INFN, Italy

Y. Guz, Y. Ivanuschenkov

IHEP-Serpukhov, Protvino, U.S.S.R.

C. Biino, S. Palestini, L. Pesando

University of Torino and INFN, Italy

R. Harr, P. Karchin

Yale University**), New Haven, Connecticut, U.S.A.

\begin{abstract}
Large aperture forward spectrometers with planar geometry perpendicular to the beam line are the natural detectors to accommodate the expected forward peaking of Beauty particle production at high energy hadron colliders. Such devices, together with silicon strip detectors for triggering, which are configured perpendicular to and close to the beam inside the vacuum pipe at the center of the interaction region should make possible the exploitation of hadron colliders as Beauty Factories. We have designed, built and run such a silicon system at the CERN Sp $\bar{p} S$-Collider. Events recorded with the RF shield of the silicon system $1.5 \mathrm{~mm}$ from the circulating beams show negligible event-unrelated background.
\end{abstract}

Submitted to Nuclear Instruments and Methods

*) Supported by U.S. National Science Foundation, Grants PHY90-08221 and PHY85-09175.

**) Supported by the U.S. Department of Energy, Contract DE-AC02-76ER-03075.

1) Ecole Polytechnique, Lausanne, Switzerland.

2) Visitor from Institute of Nuclear Physics, Krakow, Poland.

3) Now at University of Torino, Italy. 


\section{Contents}

1 Introduction 1

2 The Detector 2

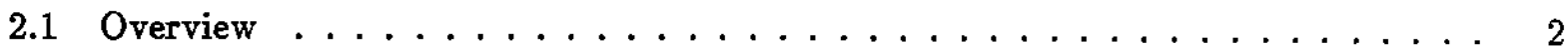

2.2 Silicon Detectors $\ldots \ldots \ldots \ldots \ldots \ldots \ldots \ldots \ldots$

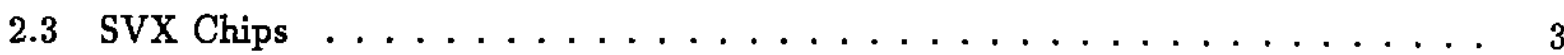

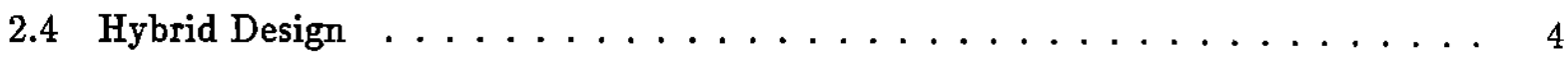

2.5 Detector Mount . . . . . . . . . . . . . . . . . . . 4

2.6 Assembly and Testing $\ldots \ldots \ldots \ldots \ldots \ldots \ldots$

3 Auxiliary Systems 5

3.1 Roman Pots . . . . . . . . . . . . . . . . . . . 5

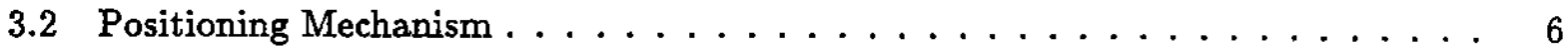

3.3 Vacuum System . . . . . . . . . . . . . . . . . 6

3.4 Silicon Power System . . . . . . . . . . . . . . . . . 7

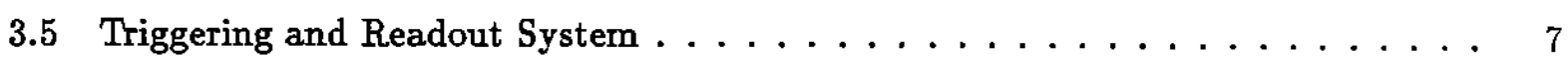

4 Results and Conclusions $\quad 8$

4.1 Operational Performance $\ldots \ldots \ldots \ldots \ldots \ldots$

4.2 Data Analysis . . . . . . . . . . . . . . . . . . 10

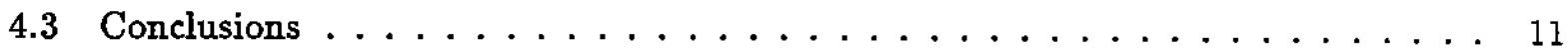

\section{Introduction}

Full exploitation of the large Beauty production cross section at hadron colliders requires a high resolution vertex detector and an efficient and highly selective topology trigger. A scheme for realizing such a detector and trigger was explored and extensively modeled in the context of a collider proposal (P238) to the CERN SPS committee[1]. It was subsequently recognized that the proposed apparatus could serve as a basis for potential Fermilab, LHC or SSC experiments with sufficient sensitivity to measure CP violation parameters in the decay of $B$ mesons.

Large aperture forward spectrometers with planar geometry perpendicular to the beam line are the natural detectors to accommodate the expected forward peaking of Beauty particle production at high energy hadron colliders. Such devices, together with silicon strip detectors for triggering, which are configured perpendicular to and close to the beam inside the vacuum pipe at the center of the interaction region should make possible the exploitation of hadron colliders as Beauty Factories.

At hadron colliders, the source distribution is a line some tens of centimeters long. Therefore, in order to minimize extrapolation distances to all possible primary vertices, the silicon detector must be positioned at the center of the interaction region, the longitudinal distance between silicon planes must be no more than a few centimeters and there must be a sufficient number of planes for complete coverage. For acceptance of small angle tracks, the gap between upper and lower halves is reduced to the minimum distance consistent with clean running conditions.

We (the P238 collaboration) have designed, built and run such a silicon system at the CERN 
Sppis-Collider. The detector is mounted in a device, called a Roman Pot, which allows retraction of the detectors to a safe distance of $5 \mathrm{cn}$ or more during beam manipulations. Background-free events are seen with the RF shield of the silicon system $1.5 \mathrm{~mm}$ from the circulating beams.

In this paper, we report on the design and construction of the detector and present a preliminary analysis of the results obtained during the Fall $1990 \mathrm{Sp} \overline{\mathrm{p} S}$-Collider run. The purposes of this test were to assess the feasibility of operating a silicon detector close to the beam axis and to gather data for the offline testing of Beauty trigger algorithms based on silicon hit information.

Fig. 1 shows side and perspective views of the detector used for this test. There are six detector planes perpendicular to the beam axis. Each plane has eight silicon detectors arranged in four quadrants. Each quadrant has independent back-to-back $\mathrm{x}$ - and $\mathrm{y}$-view strip detectors. A full 16-20 plane detector with this geometry would be optimal for particles traveling in the forward and backward directions at angles less than $600 \mathrm{mrad}$, the aperture of the proposed open geometry forward Beauty spectrometer [1].

The detector assembly and associated systems are discussed in Chapters 2 and 3 . A discussion of the performance of the detector during the collider run and a preliminary analysis of the data taken during that run is presented in Chapter 4.

\section{The Detector}

\subsection{Overview}

Fig. 2 is a photograph of a single silicon detector with its associated hybrid readout board. The assembly pictured here was designed for readout of the horizontal ( $x$-view) coordinate. The pre-amplifier/readout chips adjacent to the silicon are discussed in Section 2.3. A Kapton readout cable is shown plugged into connectors which are surface-mounted on the hybrid. The completed microvertex detector utilizes 24 assemblies of the type pictured here and another 24 assemblies, which use the same silicon detector but have a modified hybrid design for readout of the vertical ( $y$-view) coordinate. In each case, there are 896 channels per detector (seven 128-channel readout chips).

The individual detectors are supported by aluminum brackets glued to the back of the hybrids. Fig. 3 is a photograph of the lower half-detector with five of the six planes installed on a mounting plate. Each "half-plane" consists of two pairs of back-to-back $x$ - and y-view detectors mounted beside each other. Fig. 4 shows a side-view sketch of two such half-planes. Each detector is shown attached to its hybrid and mounting bracket. The longitudinal distance between paired $\mathrm{x}$ - and $\mathrm{y}$-view detectors is $2 \mathrm{~mm}$ and the distance between the center lines of adjacent planes is $3.8 \mathrm{~cm}$. Fig. 5 is a sketch of the full upper and lower detector assemblies installed at the SPS.

The thin curved line in Figs. 4 and 5 represents a $200 \mu \mathrm{m}$ thick aluminum window which serves as an RF shield against the circulating beam bunches and also isolates the detector volumes from the SPS vacuum. This shape, rather than a flat-bottomed box, was chosen in order to minimize the material close to the beams and to reduce the interactions of small angle tracks. The detector volumes were evacuated to avoid the deformation or rupture of the thin window. The silicon detector mounting plate was water cooled. It is shown attached to the vacuum bulkhead in Figs. 5 and 6.

The total distance between the outer: surface of the aluminum window and the first sensitive silicon strip in Fig. 4 is $1600 \mu \mathrm{m}$, which is the sum of the aluminum window thickness (200 $\mu \mathrm{m})$, the gap between aluminum window and silicon detector $(900 \mu \mathrm{m})$ and the dead space in the silicon $(500 \mu \mathrm{m})$. We believe that, in a future version of this type of detector, each of these components could be reduced by about a factor of two. In addition, with future use of single plane, double-sided readout detectors, the width of the window corrugations closest to the beam 
could be reduced.

\subsection{Silicon Detectors}

The silicon strip detectors were fabricated by the Central Institute for Industrial Research (S.I.) in Oslo and are functionally identical to those used by the DELPHI experiment[2]. They have an active area of $44.8 \times 44.8 \mathrm{~mm}^{2}$ and thickness $280 \mu \mathrm{m}$.

Each detector has a total of 1793 diodes, with a pitch of $25 \mu \mathrm{m}$, only half of which (every second one) is instrumented with readout electronics. The charge deposited on non-instrumented diodes capacitively induces signals on neighboring strips, resulting in improved spatial resolution at the expense of somewhat increased noise since the total charge is shared between two strips.

Metal strips, which are bonded to preamplifier inputs, run the length of and couple capacitively to the underlying diode strips via a $200 \mathrm{~nm}$ thick oxide layer. Each diode strip is connected to a common bias bus through individual $\sim 6 \mathrm{M} \Omega$ polysilicon resistors. We find a yield of more than $99 \%$ good strips and average strip leakage currents in the range of $50 \mathrm{pA}$ to $1 \mathrm{nA}$.

\subsection{SVX Chips}

A detailed discussion of the properties of the Lawrence Berkeley Laboratory designed SVX chip (we used version D) can be found elsewhere[3]. Here we give only a functional description relevant to the design of the support electronics.

The SVX is a CMOS chip which contains 128 charge-sensitive preamplifiers with an overall gain of about $15 \mathrm{mV} / \mathrm{fC}$, as well as circuitry for multiplexing and (optionally) "sparsifying" pulse-height information (i.e. reading out only those strips which have charge larger than a set threshold).

In addition to the 128 preamplifier inputs, the SVX has the following input/output lines:

- A set of eight bi-directional data lines, used to read addresses of hit strips, to set and reset various switches in the pre-amplifiers, and to write a chip identification number during initialization.

- Three control signals which determine the function of the data lines.

- A calibration line, with which charge can be injected for either diagnostic purposes or to adjust an effective threshold for sparse readout.

- Two signals ("Priority-In" and "Priority-Out") which allow daisy-chaining of an arbitrary number of SVX chips. Assertion of Priority-In enables the chip readout. Priority-Out is asserted by the chip upon completion of readout of its channels.

- A gated analog line which, during readout, outputs a voltage proportional to the charge on the strip currently being read out. The analog line is only active when the chip is enabled.

For each strip read out, the SVX outputs the chip identification number followed by the strip number onto the eight data lines, while simultaneously outputing the analog pulse height onto the gated analog line. The SVX chip requires the following voltage sources: $+6 \mathrm{~V}$ for the preamplifiers and substrate bias and $+5 \mathrm{~V}$ for the digital section. Each SVX chip requires approximately 200 mwatts. 


\subsection{Hybrid Design}

The seven SVX chips required for each detector were mounted on ceramic alumina hybrid circuit boards. Fig. 2 shows a completed hybrid on an $\mathbf{x}$-view detector. A different but similar hybrid design for the $y$-view detectors (not shown here) was needed since, due to space limitations, connections could only be made near the outer horizontal edges.

The hybrid boards were designed by our group and manufactured at the CERN Surface Treatment Workshop. Both hybrid types contain five main layers silk-screened onto the substrate. The two lowest layers have traces, which bring the control, data, calibration, and analog signals to and from the SVX chips. Two planes which provide power to the analog and digital sections of the SVX and a ground plane are layered above. Surface-mount capacitors provide power filtering. The SVX chips axe glued with a silver-impregnated epoxy onto an exposed section of the analog power plane. Connections between the input/output pads of the SVX chips and the gold traces on the hybrid are made via an ultrasonic wire bonding process using aluminum wire with 0.001 inch diameter. External connections are made via two 16-pin surface molnt connectors manufactured by DuFont (Microflex).

\subsection{Detector Mount}

The hybrids were glued, using silver-impregnated epoxy, to aluminum mounting brackets, which also served to conduct heat (approximately 1.5 watts per detector assembly) from the SVX chips to the mounting plates. The individual silicon detectors were glued to the hybrid/bracket assembly under a microscope and on a vacuum jig, with a nominal precision of $10 \mu \mathrm{m}$ perpendicular to the strips and $100 \mu \mathrm{m}$ parallel to the strips.

The detectors were positioned for gluing with reference to alignment holes on the mounting bracket and to the bracket surface which abuts the mounting plates. Assembled detectors were attached to the mounting plates with screws and held in position with alignment pins.

The two water-cooled mounting plates (for top and bottom hodoscopes) each consisted of two $2 \mathrm{~cm}$ thick plates glued together. Before gluing, a channel for cooling water was machined into the inner surface of one of the disks and matching slots were machined through both disks for the passage of readout cables. Figs. 3 and 6 are photographs of the assembly.

Each mounting plate was attached to vacuum bulkhead plates by means of four precision aluminum rods. The vacuum bulkhead plates were made from $3 \mathrm{~cm}$ thick aluminum plates, which bolted onto the Roman Pot assernbly (described below). Signals to and from the SVX chips passed from detector vacuum to atmosphere via printed-circuit Feedthrough boards which were epoxied into slits in the vacuum bulkhead plate, as seen in Fig. 6. The bulkhead plates also contained ports for the vacuum line and for cooling water.

\subsection{Assembly and Testing}

Upon receipt from the manufacturer, the silicon detector wafers were subjected to a thorough visual inspection at a microscope station in order to find physical defects such as shorts between strips, breaks in the bias and guard rings or diffusion strips, and poorly cut wafer edges.

After visual inspection, electrical measurements were made of the diode/readout-strip coupling capacitance, the interstrip capacitance and the polysilicon resistors for a random sample of strips. The leakage currents of the guard ring and bias strip were then monitored for a period of 40 to 50 hours. Typically, bias strip currents were in the range $0.1-1.0 \mu \mathrm{A}$ while the guard ring currents would increase over a period of about one day from an initial value of about $1-10 \mu \mathrm{A}$ to $20-100 \mu \mathrm{A}$ and then stabilize. Approximately $10 \%$ of the detectors were rejected either for physical or electrical defects. 
The SVX chips were tested at Lawrence Berkeley Lab using a procedure developed for the CDF microvertex detector. Using the test results, an attempt was made to match as closely as possible the nominal analog output voltage of the seven SVX chips on a given detector. No additional tests were performed on the SVX chips before they were mounted on the hybrid assembly.

After manufacture, the ceramic hybrid boards were visually inspected for obvious defects. The passive components (resistors, capacitors and connectors) were then mounted. The hybrids were tested for continuity between bonding pads and connectors and for shorts between adjacent lines. The SVX chips were then glued in place and wire-bonded to the hybrids.

At this point, the hybrid assembly was tested. The test setup was similar to the experimental readout system which is discussed in Sec. 3.5. It consisted of a MicroVax computer controlling a CAMAC crate which contained a sequencer module (SRS)[4] to generate the SVX clocking signals and a readout module (SDA)[4] to read and store the SVX analog and digital address information. A test board provided the electrical interface to the detector assembly and contained a variable charge injection circuit as well as oscilloscope test points. The following requirements were made:

- analog output voltage responds to charge injected via the calibration line;

- Readout Priority signal propagates through the SVX chain;

- fewer than $3 \%$ of the channels have average analog outputs outside specified limits;

- fewer than $3 \%$ of the channels have unacceptably high noise levels;

- no (or few) skipped, out of sequence, or multiple occurrences of digital addresses.

Problems detected by the testing were usually repaired. However, not all problems were considered serious enough to warrant the risk of creating a worse condition by attempting repairs.

After testing, the hybrid, mounting bracket and detector were assembled and the 896 readout strips were bonded to the inputs of the SVX preamplifiers using a semi-automatic bonding machine, which required a few hours per detector. The above tests were then repeated. As a final test, each detector was scanned with a tightly focused, pulsed LED light source to verify that all (or almost all) channels responded and to get a rough measure of the relative analog gain of the seven SVX chips. The detectors were then fixed to the mounting plates, as shown in Fig. 3.

\section{$3 \quad$ Auxiliary Systems}

\subsection{Roman Pots}

The silicon detector was isolated from the $\mathrm{Sp} \overline{\mathrm{p}} \mathrm{S}$ vacuum for two reasons:

1. The vacuum pressure in experimental areas of the $\mathrm{Sp} \overline{\mathrm{p}} \mathrm{S}$ must not exceed about $5 \times 10^{-10}$ mbar and therefore extreme care must be taken in the use of materials and feedthroughs. Placing the silicon detectors in a secondary vacuum volume minimizes the outgassing problems connected with the choice of detector materials and the leakage requirements of feedthroughs for the detector signals and power.

2. The detector must be shielded from the electromagnetic fields accompanying the passage of beam bunches which could potentially induce large signals. 
Thus, a conductive vacuum-tight separation vessel with low outgassing rate was needed. The window of this vessel, through which particles pass, should offer minimal material for multiple scattering, particularly to small angle tracks. We therefore developed a special aluminium vacuum vessel which had a corrugated $200 \mu \mathrm{m}$ aluminum foil window as shown in Figs. 4 and 5. Since such a thin window could not withstand the pressure difference of one atmosphere, the silicon detector assembly volume was pumped down to about $10^{-4}$ mbar.

Fig. 7 shows the aluminum vacuum vessel (Roman pot) with its two corrugated side walls before attachment of the thin window. This piece was machined from a single block of aluminum using a computer-controlled milling machine. The free space between the two side walls at the rectangular opening, through which the silicon detector planes protrude, was covered with the $200 \mu \mathrm{m}$ thick aluminum foil.

The corrugated shape of the aluminum window was produced using the special tool shown in Fig. 8 and was afterwards annealed in order to stabilize the corrugated shape. The window was glued to the side walls of the pot with a silver-impregnated epoxy to ensure good electromagnetic shielding. Fig. 9 shows a close-up of the corrugated region of the pot, after installation of the aluminum window.

Since the total surface of glue ( $\sim 50 \mu \mathrm{m}$ thick line over the length of the corrugated shape) exposed to the Ultra High Vacuum (UHV) system of the SPS is less than $1 \%$ of the surface of the complete pot, its contribution to the total outgassing rate is negligible. Fig. 10 shows a detail of the connection of the aluminum window to the pot.

\subsection{Positioning Mechanism}

Given the short time allowed for construction of equipment for this test, it was not possible to design and build an optimized positioning mechanism. We therefore used, with minimal compromise, an existing Roman Pot mechanism[5] which had previously been used by the UA4 and UA8 experiments at the Spp̄S-collider. Fig. 11 is a photograph of the pot mechanism installed at the SPS.

The mechanism allows detectors, mounted inside pots above and below the beam, to be displaced along the vertical axis. The pot flanges are connected to the vacuum chamber of the machine by bellows. During beam manipulations, the edge of the RF shield was retracted to a distance of about $5 \mathrm{~cm}$ from the nominal beam position. During data-taking runs, this edge was brought to a distance of $1.5 \mathrm{~mm}$ from the beam axis, as sketched in Fig. 5. The pot position was adjusted and monitored using a linear position transducer with an accuracy better than $50 \mu \mathrm{m}$.

\subsection{Vacuum System}

Fig. 12 shows the schematic layout of the special pumping system installed for this test. Initial pump-down of the SPS vacuum system and pots from atmospheric pressure to about $10^{-1}$ mbar is made with the turbomolecular pump VPRS1, via the valves, $\mathrm{V} 1$ and $\mathrm{V} 2$. Below a pressure of $10^{-1}$ mbar, as measured on gauges VG2 and VG-SPS, valve V2 closes automatically and VPRS1 pumps only the pots via V1. At the same time valves V3 and V4 are opened to pump the SPS vacuum system with the help of turbomolecular pumps VPRS2 and VPRS3. After a bakeout at $150^{\circ} \mathrm{C}$ of the SPS vacuum system, ultra high vacuum production is started with the VPS pumps (combination of a $400 \ell / \mathrm{s}$ sputter-ion pump with a $1000 \ell / \mathrm{s}$ sublimation pump).

In case of a sudden pressure rise of more than $10^{-1} \mathrm{mbar}$ in the SPS vacuum system or in the Roman Pots, SPS sector valves VVS1 and VVS2, as well as the roughing valve V1, close and the separation valve V2 opens. This equalizes the pressures in both vacuum systems and therefore avoids damage to the detectors and thin windows of the pots. 
After a short power cut, the pumping system restarts automatically. However, in the event of an extended power failure, resulting in a pressure rise above $10^{-1} \mathrm{mbar}$, the entire pumping system is blocked and, for safety reasons, can only be restarted manually.

The best pressure obtained in the SPS vacuum system after a few weeks of pumping was about $5 \times 10^{-10} \mathrm{mbar}$ with the residual gas mainly composed of hydrogen and water vapor. The pressure in the pots was maintained at about $5 \times 10^{-4}$ mbar. The gas load of the complete silicon detector assembly was about $\left(\sim 10^{-3} \mathrm{mbar} * \ell / \mathrm{s}\right)$.

\subsection{Silicon Power System}

The power system supplies DC voltages to run the detectors, with the requirements that these voltages have low noise $(<1 \mathrm{mV}$ at the detector) and that every bias current is monitored. The system consists of: (i) a switch panel in the counting room for individual remote control of each of the 48 detectors; (ii) power supplies mounted in a rack next to the detector; (iii) monitoring system consisting of a CAMAC multiplexer and ADC module, read out by a Caviar[6] microprocessor unit in the counting room. The use of opto-couplers to switch individual detectors assures total electrical isolation of the control circuitry thus minimizing noise pick-up.

The SVX analog and digital voltages as well as voltages to power the external electronics (described below) are generated by commercial supplies (Ultronix).

Batteries are used to bias the detector back planes, since suitably decoupled batteries provide ripple-free voltage, and make individual detector current monitoring relatively simple. Four 90 volt batteries (Eveready No. 490) are mounted in a double-width NIM module. The output voltage for each channel can be independently adjusted in the range 0 to $90 \mathrm{~V}$ and is determined by a combination of an external reference voltage, common to all supplies and potentiometers for each channel, as shown in Fig. 13. Two Darlington-connected transistors provide a low output impedance, and immunity from battery voltage decay. With typical bias voltages of $50 \mathrm{~V}$ and currents of 1 to $100 \mu \mathrm{A}$ (due mainly to guard ring leakage), battery life is greater than 6 months.

The voltages and currents of all supplies are continuously monitored by a CAMAC analog scanner and DVM (Lecroy LG5310) and displayed in the counting room. The SVX analog and digital voltages and the detector bias voltage are interlocked to insure that they could be only turned on in the correct order.

\subsection{Triggering and Readout System}

The detector readout was triggered by an arm-arm coincidence of two scintillation counters, which detected particles produced at polar angles between 27 and 110 mrads. According to Monte Carlo calculation, the trigger was sensitive to about $45 \%$ of the total cross section, thus giving us an approximate minimum bias data sample.

Control signals for operation of the SVX chips were generated by the Lawrence Berkeley Laboratory SRS (SVX Readout Sequencer)[4] CAMAC module. This module contains an AMD2910 sequencer and 12 kbytes of program memory which may be downloaded from the host computer. The 6-byte instruction length uses 2 bytes for the AMD2910 instruction-code and 4 bytes for bit patterns, which are output via a front panel connector to the SVX data and control lines.

A typical program contains a block of instructions for setting up a sample-and-hold sequence and another block containing instructions for readout control. The state of front panel input signals (e.g. a scintillator trigger signal) can be used to modify program flow with a conditional branch instruction.

The SRS control signals were converted to differential levels and sent over a $50 \mathrm{~m}$ flat twisted pair cable to the interaction region, where they were converted to single-ended TTL levels. The 
single-ended control signals were then bussed over two flat cables to the 12 Plug-In-Driver (PID) printed circuit boards, which were mounted on the exteriors of the upper and lower pot mechanisms. As sketched in Fig. 14 for one detector half-plane, the PID boards plug into Feedthrough printed circuit boards, which pass signals through the vacuum bulkhead to and from the detector hybrids via short Kapton cables. to:

The purpose of the PID boards, each of which serviced the 4 detectors in one half plane, was

- fan out control and data signals to the hybrids;

- connect the analog lines of paired $x$ - and $y$-view detectors from each quadrant to produce two $x / y$ daisy-chains of 1792 channels per PID board;

- convert the single-ended SVX analog output signals to differential levels which are sent to the counting room via $50 \mathrm{~m}$ of shielded twisted pair cables;

- multiplex the digital data signals (strip number and chip number) from the SVX chips onto a bus common to all PID boards. Signals from any daisy-chain could be selected from the counting room for diagnostic purposes. (This bus is not shown in Fig. 14.)

The SRS was synchronized with the beam bunch crossings by means of a signal from a directional coupler which sensed the bunch passages. When live, the SRS generated a sampleand-hold sequence for every beam bunch crossing $(3.8 \mu \mathrm{sec})$. Upon receipt of a scintillator trigger, the SRS initiated and controlled the SVX readout.

We used the CERN-designed SIROCCO I[7] (SDicon ReadOut Camac COntroller) module for analog to digital conversion. This module features optional hit sparsification as well as pedestal subtraction. Baseline offsets in the SIROCCO analog section were cancelled between readouts, which reduced our common-mode noise (see discussion in Section 4.1). The SIROCCO module contains a 10-bit flash ADC and internal memories to store pedestals and pedestalsubtracted pulse heights for up to 2048 channels. 24 SIROCCO modules were used, one for each daisy chain.

Readout to the host computer (MicroVAX II) was implemented using standard CAMAC and CERN ROMULUS/REMUS[8] modules. Silicon hit data together with pulse-height, pulse arrival time and scaler information from the scintillators were written to $8 \mathrm{~mm}$ cassettes using Exabyte tape drives.

\section{Results and Conclusions}

\subsection{Operational Performance}

The silicon Microvertex detector was run in Interaction Region LSS5 at the CERN Spp̄SCollider $(\sqrt{s}=630 \mathrm{GeV})$ from September through December 1990 with peak luminosities of $3 \times 10^{30} \mathrm{~cm}^{-2} \mathrm{~s}^{-1}$.

A total of about 8.6 million minimum bias events were written to tape using either full detector readout or the SIROCCO sparsification feature. 1.6 million of these were written in the full readout mode, where the event size was $172 \mathrm{kbytes}$ and the data acquisition rate was limited to about $3 \mathrm{~Hz}$ by the tape writing speed.

About 7 million events were recorded using the readout sparsification mode. For each SIROCCO module, the threshold was set at three times the average r.m.s. noise found in the two daisy-chained detectors read out by that module. Only channels passing the threshold were read out and written to tape. In order to monitor pedestal stability and common mode 
noise, 21 additional "monitor" channels ( $2 \%$ of the total) were always read out. Thresholds for "hot" channels were set such that those channels were never read out. The event size was typically 9 kbytes, and the acquisition rate was limited by computer response time to about 35 Hz.

No increases in scintillator counting rates were seen whenever the pot positions were manipulated, for example when the pot window to beam distance was decreased from 50 to $1.5 \mathrm{~mm}$ (corresponding to 15 r.m.s. beam widths). This indicated that the beam scrapers, which were positioned at 6 r.m.s. beam widths, were still effectively shadowing the detector assembly. ${ }^{1}$ In this position, the first sensitive silicon strip was $3.1 \mathrm{~mm}$ from the circulating beams.

No evidence was seen for run-to-run variations in the average vertical position of the beams and thus, under normal running conditions, we could set the detectors to $1.5 \mathrm{~mm}$ from the beam line at the start of every $\mathrm{S} p \overline{\mathrm{p}} \mathrm{S}$ fill. Preliminary analysis of reconstructed vertices shows that over the course of a run, the position of the Spps beams varied by less than $100 \mu \mathrm{m}$ in both the vertical or horizontal directions, with respect to our detectors.

The silicon detectors were found to function well under vacuum in the Roman Pots. Total detector leakage currents were found to decrease when a detector was operated in vacuum and remained low during the course of a run. This behavior is presumably due to the decrease of surface moisture in vacuum. Typical total leakage currents varied between 1 and $20 \mu \mathrm{A}$ while in vacuum. This compares to values in the range of 100 to $200 \mu \mathrm{A}$, as measured on the test bench in air.

One detector out of the 48 failed because its back-plane developed a short to ground during installation. Two SVX chips (256 channels) of another detector failed during the run due to an accident. Of the remaining 46 detectors, 407 channels( $1 \%$ of remaining channels) were unusable due to high noise levels caused mainly by defects in the silicon (e.g. holes in the $\mathrm{SiO}_{2}$ layer resulting in shorts between a diode and its readout strip). Therefore, a total of $96.4 \%$ of the 43008-strip system functioned correctly.

For the 1.6 million events with full analog readout, the pedestal value of each channel was determined offline from the mean pulse height in the first 100 events on each tape, after deleting hit candidates. "Common mode" noise (event-to-event fluctuation of the pulse height of every channel in a given SVX chip by the same amount) was found to be typically a few ADC counts. To remove this noise, the average pedestal-subtracted pulse height of the 128 channels in each SVX chip for a given event was subtracted from the pulse height in each channel before hit-finding (the same subtraction was also done during pedestal finding). The r.m.s. noise, averaged over the 100 events, after pedestal and common mode noise subtraction, is shown for each channel of a typical detector in Fig. 15(a) and is typically $3.4 \mathrm{ADC}$ counts. For the 7 million events recorded with sparse readout mode, the monitor channels were used to determine the common mode correction for each event.

The r.m.s. single-channel noise showed no noticeable increase during the course of the collider period. Indeed, the data shown in Fig. 15(a) were taken near the end of the run. Since the beam passed closest to strip 896 , the radiation dose from interactions increased with increasing strip number. The fact that the r.m.s. noise shows no corresponding increase indicates that no significant interaction-induced radiation damage had occurred (this is not surprising, since the integrated luminosity for our entire run was about 2 inverse picobarns). Dosimeters were mounted in the Roman Pot near the detector, but detailed analysis of accumulated dose rates awaits dismantling of the detector.

We looked for evidence of RF noise pickup from the circulating beams. However, no differences were found either in the pedestal values or widths, with and without beam present. Thus,

\footnotetext{
${ }^{2}$ Near the end of one run, when the circulating beams were larger, we were permitted to move one pot in to a distance of $1.2 \mathrm{~mm}$ from the beam. The scintillator counting rates increased by a factor of about two. This increase can be explained if approximately one halo track per bunch crossing traversed the aluminum shield.
} 
the RF shielding provided by the aluminum window was adequate.

The signal from a single charged particle is usually spread over more than one strip for two reasons. For tracks at normal inciclence, a combination of charge diffusion and capacitive coupling of adjacent strips usually results in a cluster width of 2 strips and, for trajectories at a large enough angle with respect to the plane normal, charge is deposited under a larger number of strips. A cluster is thus defined as a contiguous series of strips, each with a pulse height more than 3 times the r.m.s. noise and with total cluster pulse height more than 0.3 times that expected for a minimum ionizing track.

The cluster pulse height distributions for a typical detector are plotted in Fig. 15(b). The average pulse heights for clusters with width 2 strips and for clusters with width less than 4 strips are 111.3 and 145.5 ADC counts, respectively. For the dominant 2 -strip clusters, this implies an average signal to noise ratio of $23[111.3 /(\sqrt{2} \times 3.4)]$. This excellent signal to noise characteristic is mainly due to the low noise of the SVX and the low input capacitance of our relatively small silicon detectors.

The most important result from the test run is that we routinely operated the silicon detector at a distance of $1.5 \mathrm{~mm}$ from the circulating beams and observed background-free events.

\subsection{Data Analysis}

The distribution of cluster multiplicity in one typical detector is shown in Fig. 16(a). In all detectors, the observed average value is 2.2. The Monte Carlo results in the figure were obtained using PYTHIA 5.3[9] for minimum bias event generation and GEANT3[10] for detailed simulation of our silicon detector system. Apart from an excess of data events with no clusters, the data and Monte Carlo agree reasonably well (possible sources of the excess zero-cluster events are under investigation). The distribution of hits as a function of strip number is shown in Fig. 16(b). The good agreement between data and Monte Carlo is a first indication that the Monte Carlo event generator is a reliable representation of the data and also that almost all clusters are caused by event-associated charged particles.

A preliminary version of the pattern recognition program finds tracks initially by pairing all clusters found in planes $\mathrm{n}$ and $\mathrm{n}+2$ and looking for associated clusters within a road width of $\pm 125 \mu \mathrm{m}$, in all other planes. On each accepted track, at most one plane which is geometrically traversed by the track is allowed to have a missed cluster.

Vertices are found by first extrapolating all $x-z$ and $y-z$ track projections to the beam line, which is constrained by the alignment procedure to lie along the $\mathrm{z}$ axis. The peak found in a histogram of $z$-intercept values is taken as the first estimate for the vertex $z$ value. The program subsequently searches for 2-point tracks which point to the estimated vertex. All track projections which have impact parameters less than $1 \mathrm{~mm}$ relative to this preliminary vertex estimate are used to find the final vertex coordinates by minimizing a $\chi^{2}$ calculated as the weighted sum of the squares of the projected impact parameters (the weight of a track is calculated from a momentum, estimated from the tracks angle, and from its fitting errors, added in quadrature). In the minimization, the $\mathrm{x}, \mathrm{y}$ and $\mathrm{z}$ vertex positions are free parameters.

Typical event displays are "clean"; i.e., there are few clusters which are not associated with tracks from a common vertex. For example, Figs. $17 \& 18$ show the $x-z$ (top view) and $y$ $z$ (side view) projections, respectively, of a typical high multiplicity event with a vertex near one end of the silicon system, such that most tracks have at least five measured points, which would be typical of a larger system with 15 or more planes. The straight lines shown represent all tracks found by the pattern recognition program. The number of tracks which come from sources outside the interaction region is negligible, less than one halo track per 1000 events. Fig. 19 contains the top view of an event which occurs at the center of the silicon system and demonstrates that, in a collider application, the geometric acceptance covers both outgoing 
hemispheres.

Fig. 20 shows the observed distribution of the number of found $x-z$ and $y-z$ track projections and its Monte Carlo expectation. Events are excluded from this plot (approximately 10\%) if they contain 10 or more tracks which have projected impact parameters relative to the primary vertex of $1 \mathrm{~mm}$ or more. Such events are dominated by multiple interactions in a bunch crossing (pileup). The small differences between data and Monte Carlo can be due to several things, such as a pileup component surviving the 10 track cut, minor inadequacies of the Monte Carlo event generator and/or accidental triggers.

Fig. 21(a) shows a cluster residual plot for one detector obtained by taking the difference between cluster centroids and the linear interpolation of cluster positions in the two adjacent detectors for clusters on all found tracks. Fig. 21(b) shows the residual distribution for tracks with slopes less than 0.1 and track $\chi^{2}$ per degree of freedom of less than 7 . These tracks tend to have higher momenta and therefore less multiple scattering. The r.m.s. resolution of $7.2 \mu \mathrm{m}$ implies a point resolution of less than $6 \mu \mathrm{m}$ for high momentum tracks.

A first look at our expected resolution in vertex separation can be obtained by examining the distribution in projected impact parameter for all tracks in events with a single vertex. Fig. 22 is the distribution of vertical or $y$-coordinate of found interaction vertices in 10,000 events with a fitted r.m.s. of $92 \mu \mathrm{m}$. Fig. 23 shows an impact parameter distribution only for tracks in a given quadrant of the silicon detector. The non-Gaussian tail is due primarily to low momentum tracks, tracks with some wrong hits or completely fake tracks. The expected distribution from our Monte Carlo simulation is superimposed on the data and agrees reasonably well, with FWHM of $90 \mu \mathrm{m}$. The small offset between data and Monte Carlo is probably due to residual alignment errors and is under study.

In a future full experiment using a silicon detector of the type discussed here, the vertex resolution will be significantly improved by the use of double-sided silicon detectors, which will halve the necessary number of silicon planes, and a thinner (i.e. $100 \mu \mathrm{m}$ ) aluminum window. Correct momentum weighting in offline vertex fits will add to the improvement.

An important use of our data is to test the proposed trigger algorithm[1] which vetoes events which satisfy a single vertex hypothesis (rather than attempt a full online multi-vertex reconstruction). In this algorithm, a $\chi^{2}$ is formed for the hypothesis that all impact parameter projections are zero, summing independently over $x-z$ and $y-z$ track projections. As above, a new vertex point is then found which minimizes the $\chi^{2}$. If the $\chi^{2} /($ degree of freedom) is smaller than some cut value the event is rejected. If larger, an iteration process is carried out, in which as many as two tracks with worst $\chi^{2}$ contribution are rejected. Events which then remain in poor agreement with the single vertex hypothesis are retained. The "trigger efficiency" versus $\chi^{2}$-cut for both our data and the Monte Carlo simulation data are compared in Fig. 24 and are seen to be in good agreement, thus again validating the correctness of our Monte Carlo simulation software. Note that the particular shape and magnitude of the distribution in Fig. 24 are characteristic of our (truncated) 6-plane system.

\subsection{Conclusions}

A large silicon system was constructed and operated successfully in close proximity to the Sppis-collider beams. The detector performance was satisfactory, with signal to noise ratios in the range 20 to 30 , which allows an online pedestal subtraction and zero suppression with high efficiency. The background due to beam halo particles, and the effect of RF induced signals from bunch crossings, was found to be negligible.

A complete detector Monte Carlo simulation using PYTHIA and GEANT has been carried out. Data and Monte Carlo have been compared and are seen to be in good agreement, a fact which validates our trigger Monte Carlo simulation calculations. The success of this test 
a)
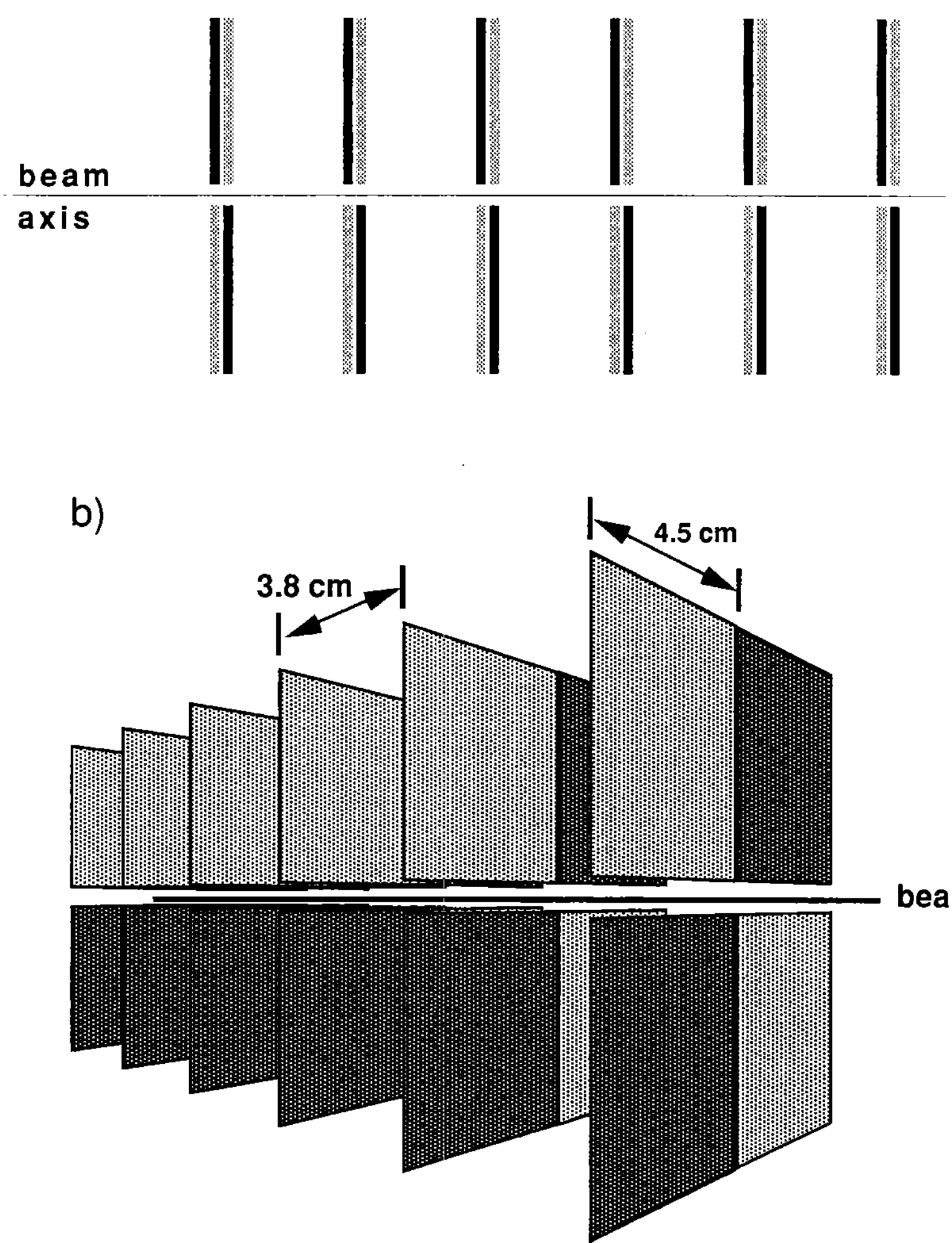

beam axis

Figure 1: (a) Side view and (b) perspective drawing of the silicon detector configuration. The 48 detectors are organized into 6 planes perpendicular to the beam axis. Each plane is divided into four quadrants and two views. A vertical gap of a few millimeters is left to allow passage of the circulating beams. In (a), the black lines indicate the $x$-view detector positions and the gray lines indicate the $y$-view detector positions. In (b), only the frontmost detectors in each plane are visible. 


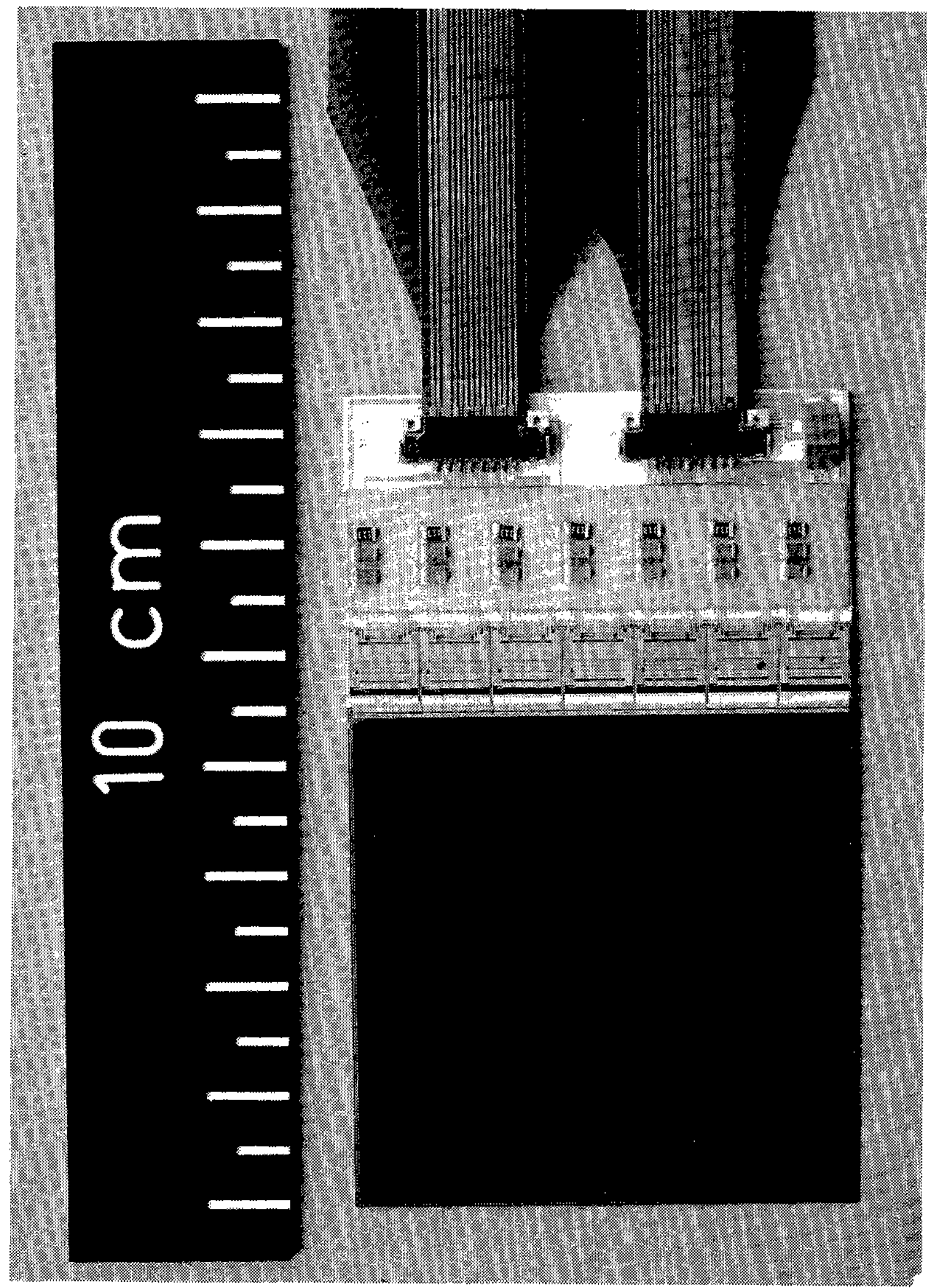

Figure 2: Photograph of a single silicon strip detector assembly for horizontal (x-view) readout. The silicon detector is the large dark square; its readout hybrid is bonded to its upper edge. Seven SVX readout chips (see Sect. 2.3) are in a row near the middle of the photograph. A Kapton readout cable enters from the top and plugs into surface-mount connectors on the readout hybrid. 


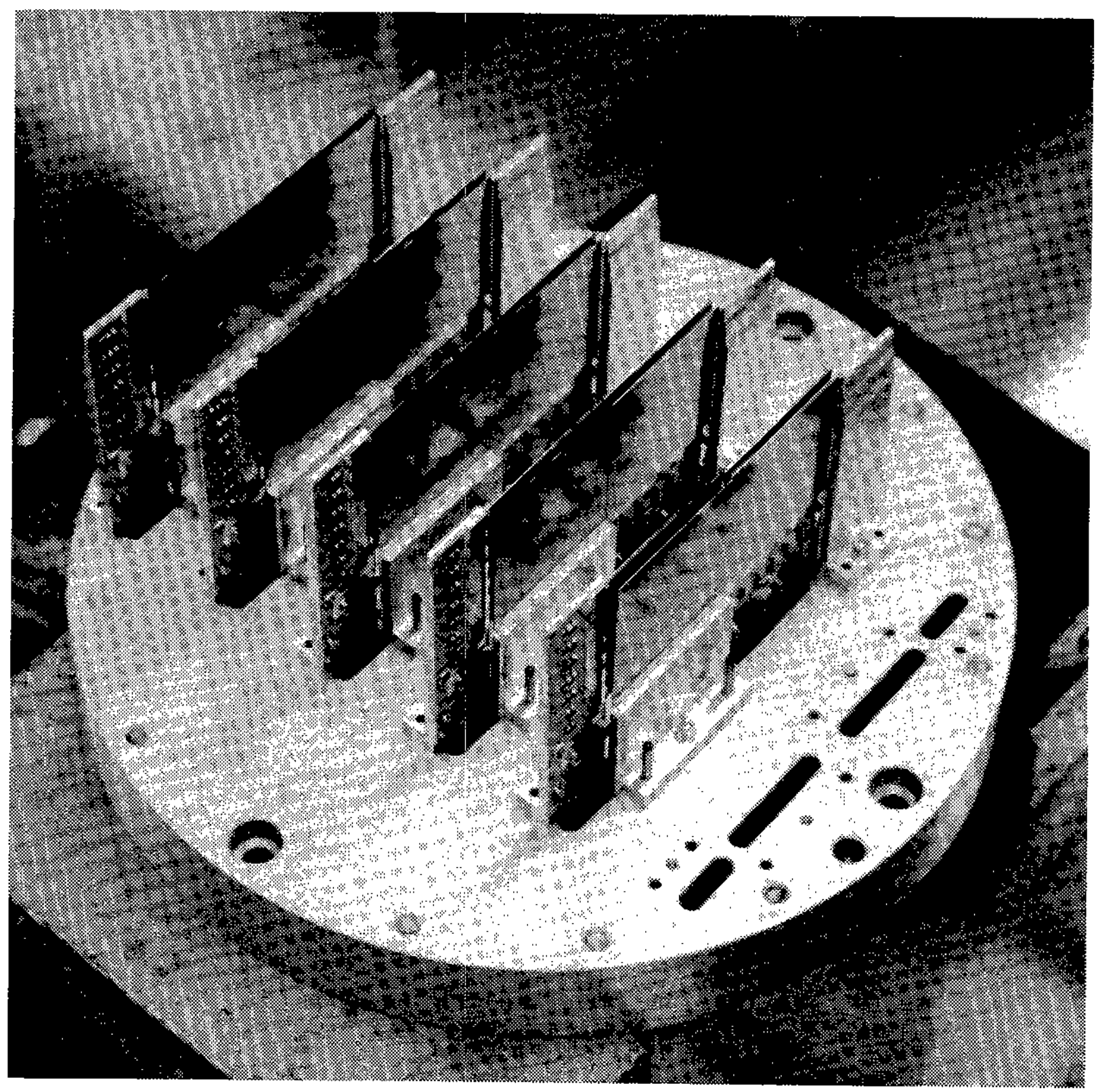

Figure 3: Photograph of a partially assembled half-detector. Five of the six planes are seen attached to the mounting plate. Cable slots for the sixth (as yet unmounted) plane are visible on the right-hand side of this photograph. Each "half-plane" consists of two pairs of back-toback $x$ - and $y$-view detectors mounted beside each other. The $2 \mathrm{~mm}$ longitudinal separation of $\mathrm{x}$-view and $\mathrm{y}$-view detectors can be seen. 


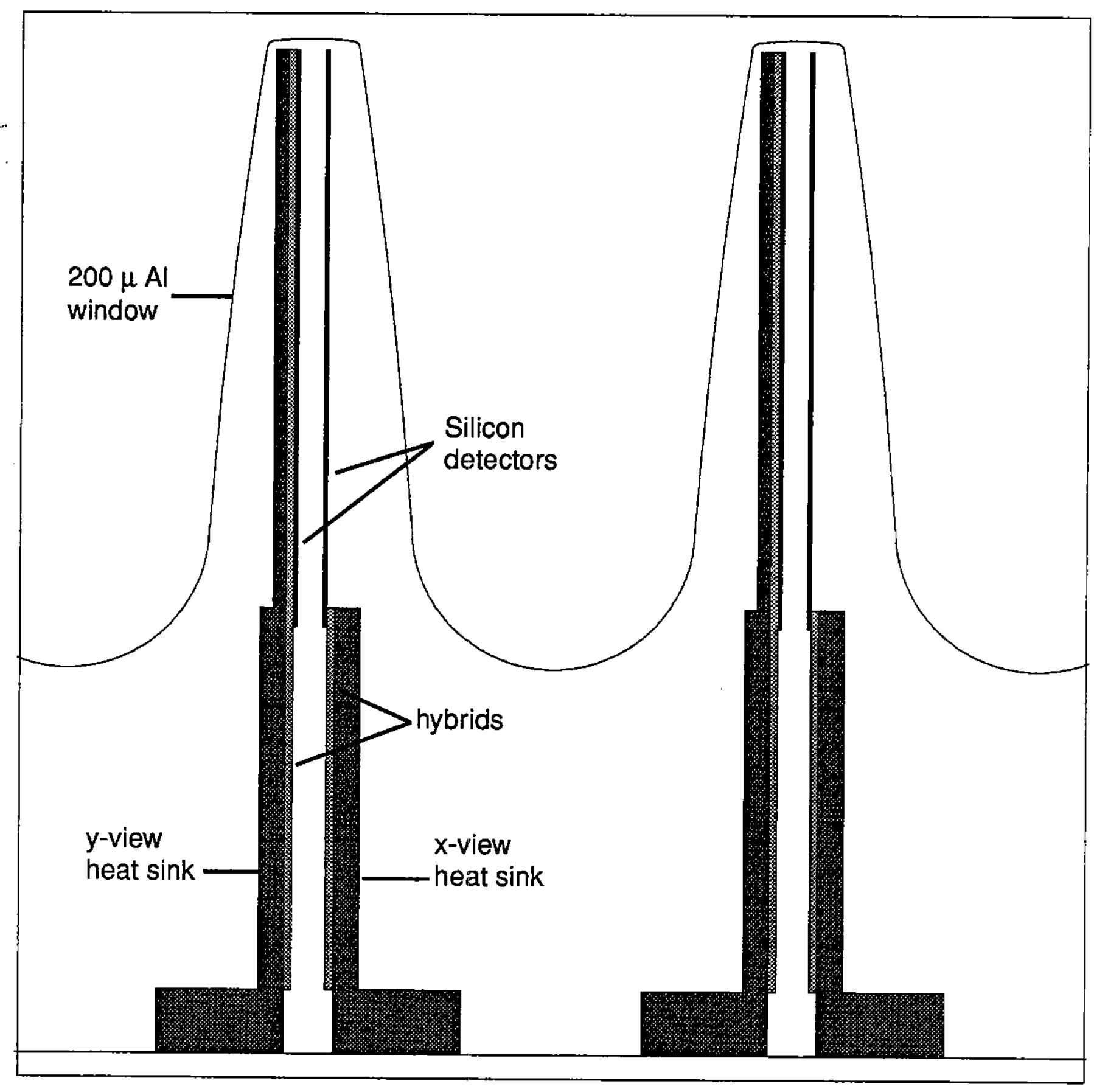

Figure 4: Side view showing two planes in the lower half-detector. The y-view detectors for each plane are on the left of the corresponding $\mathbf{x}$-view detectors. The readout hybrids are glued to aluminum brackets which are attached to the water-cooled mounting plate near the bottom of the sketch. The curved line is the $200 \mu \mathrm{m}$ aluminum window. 


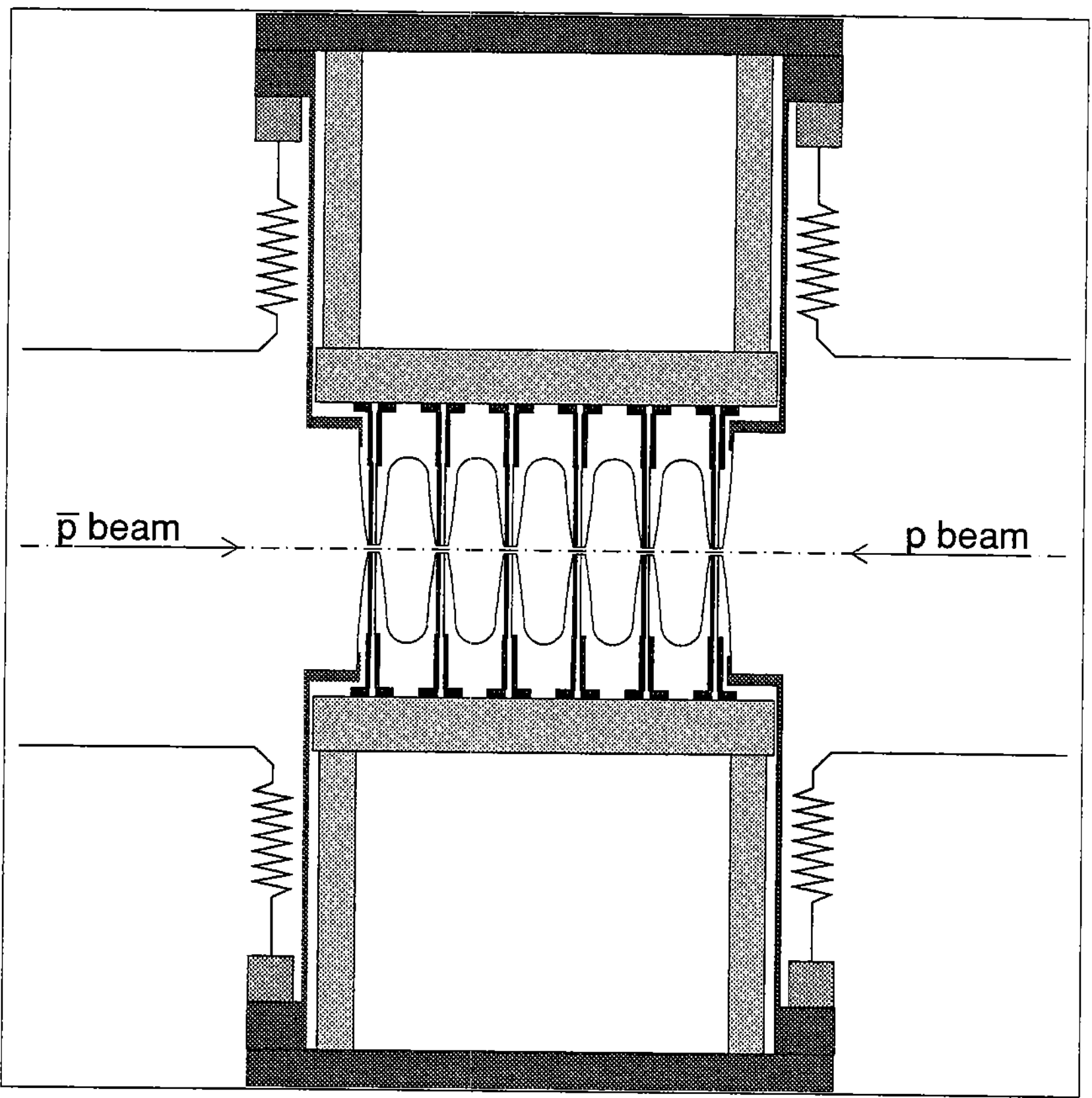

Figure 5: Sketch of the full detector assembly and Roman Pots. The inner edges of the aluminum windows are at a distance of $1.5 \mathrm{~mm}$ from the beams. The detector mounting plates are attached to the vacuum bulkheads (outermost dark areas) via four vertical rods (two of which are shown). The vacuum bulkheads are bolted to the Roman Pot positioning mechanisms. The bellows (zigzag lines) allow pot movements in the vertical direction. 


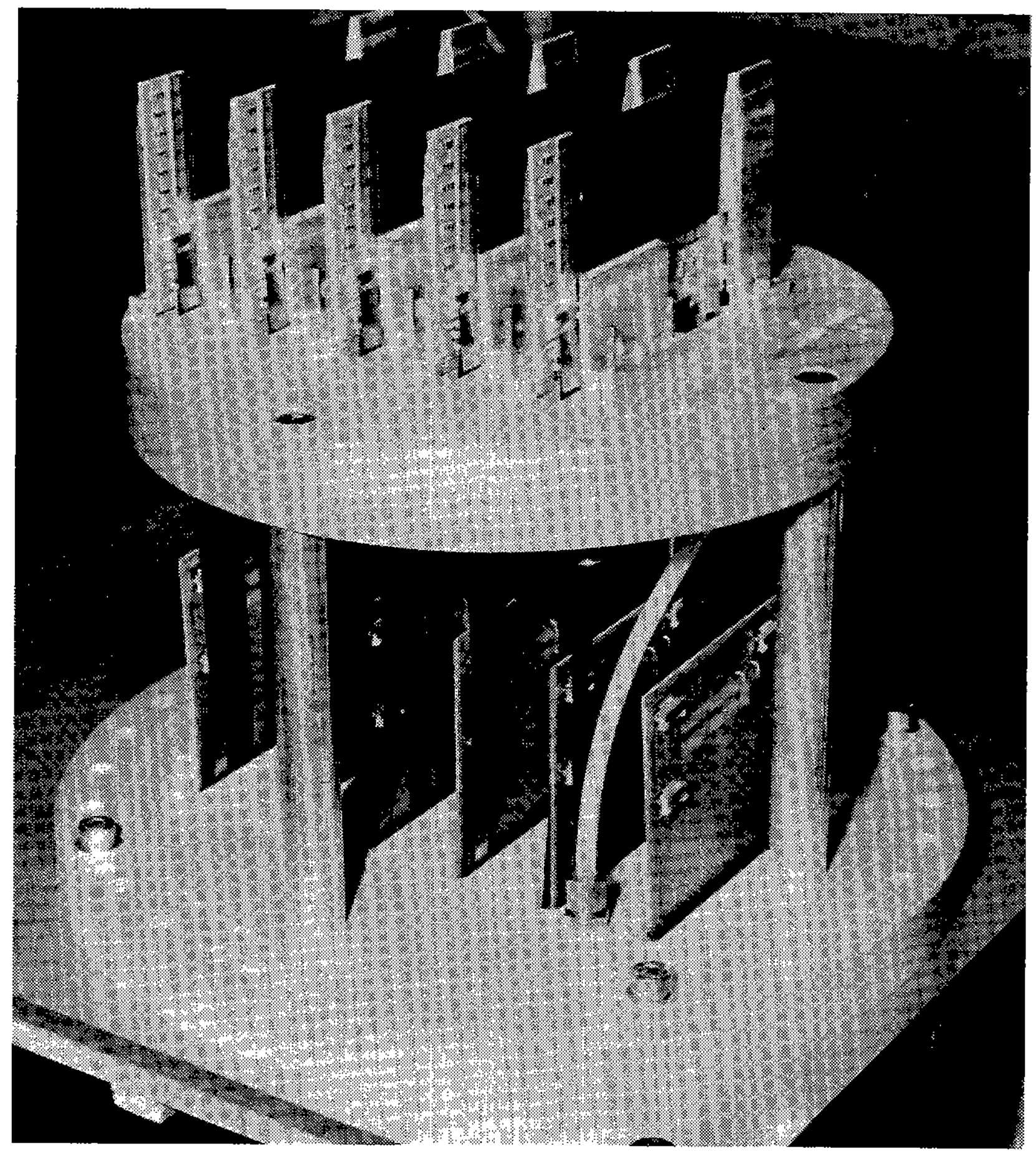

Figure 6: Photograph of the five-plane assembly of Fig. 3, joined to a vacuum bulkhead. The bulkhead is at the bottom of the figure. The vacuum-side portions of the Feedthrough boards emerge through the slits in the vacuum bulkhead plate. A cooling-water tube is visible in the foreground. Two of the four rods which fix the mounting plate to the bulkhead are visible. 


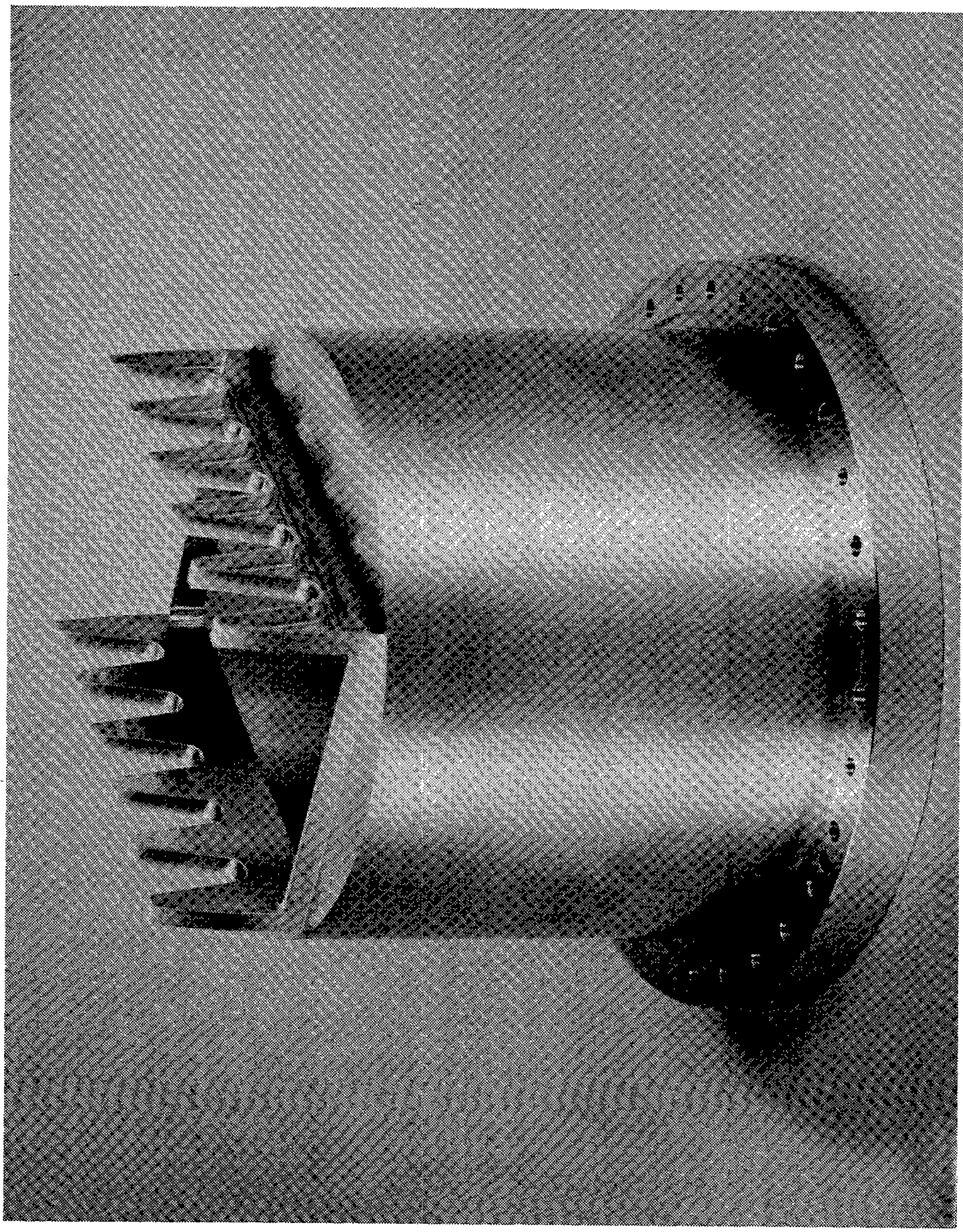

Figure 7: Photograph of one of the aluninum vacuum vessels (Roman Pot) before attachment of the aluminum window. This piece was machined from a single block of aluminum. The detector assembly is inserted through the bottom of this piece with detector planes emerging in the corrugated region near the top of the photograph. 


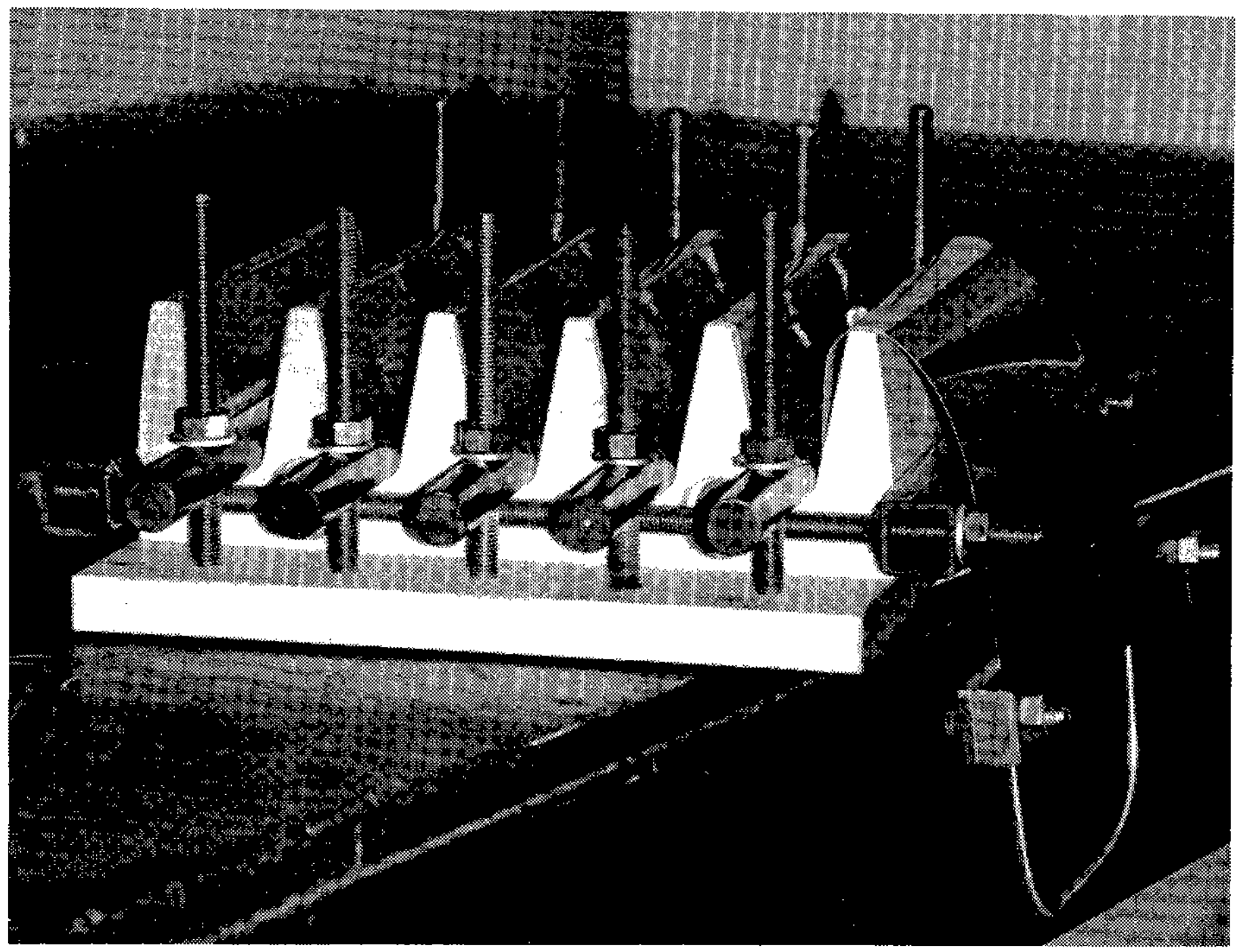

Figure 8: Jig used for pre-forming the $200 \mu \mathrm{m}$ aluminum window. The window sheet is first clamped to the left-hand side of the jig and then forced to follow the contours of the six "teeth". The teeth were machined to the shape of the side walls of the Roman pot shown in Fig. 7. 


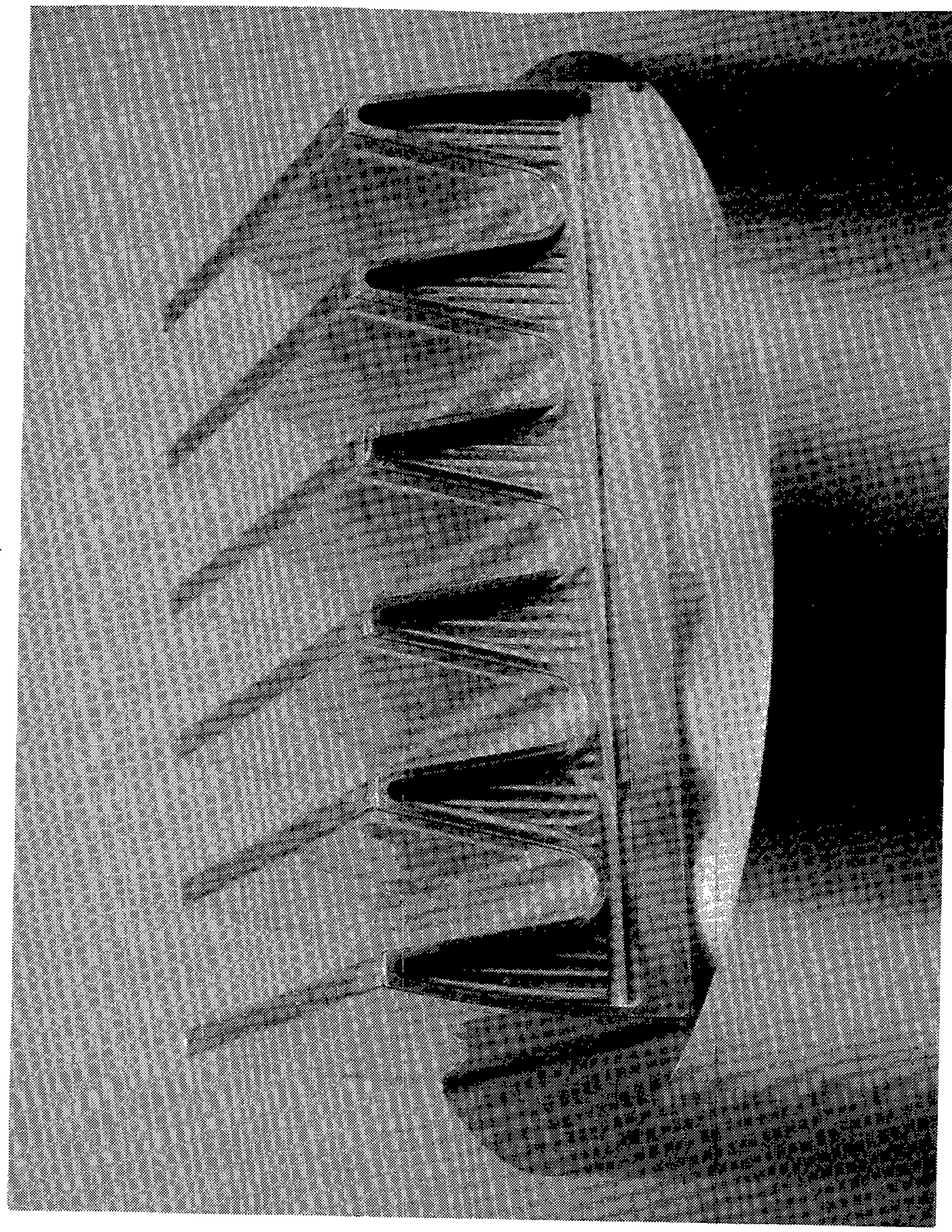

Figure 9: Close-up photograph of the top of a completed Roman Pot showing the corrugated aluminum window. During data taking, the circulating beams were normally $1.5 \mathrm{~mm}$ above the flat edges of the protruding window sections. The silicon planes are positioned inside the windows, as sketched in Fig. 4. 


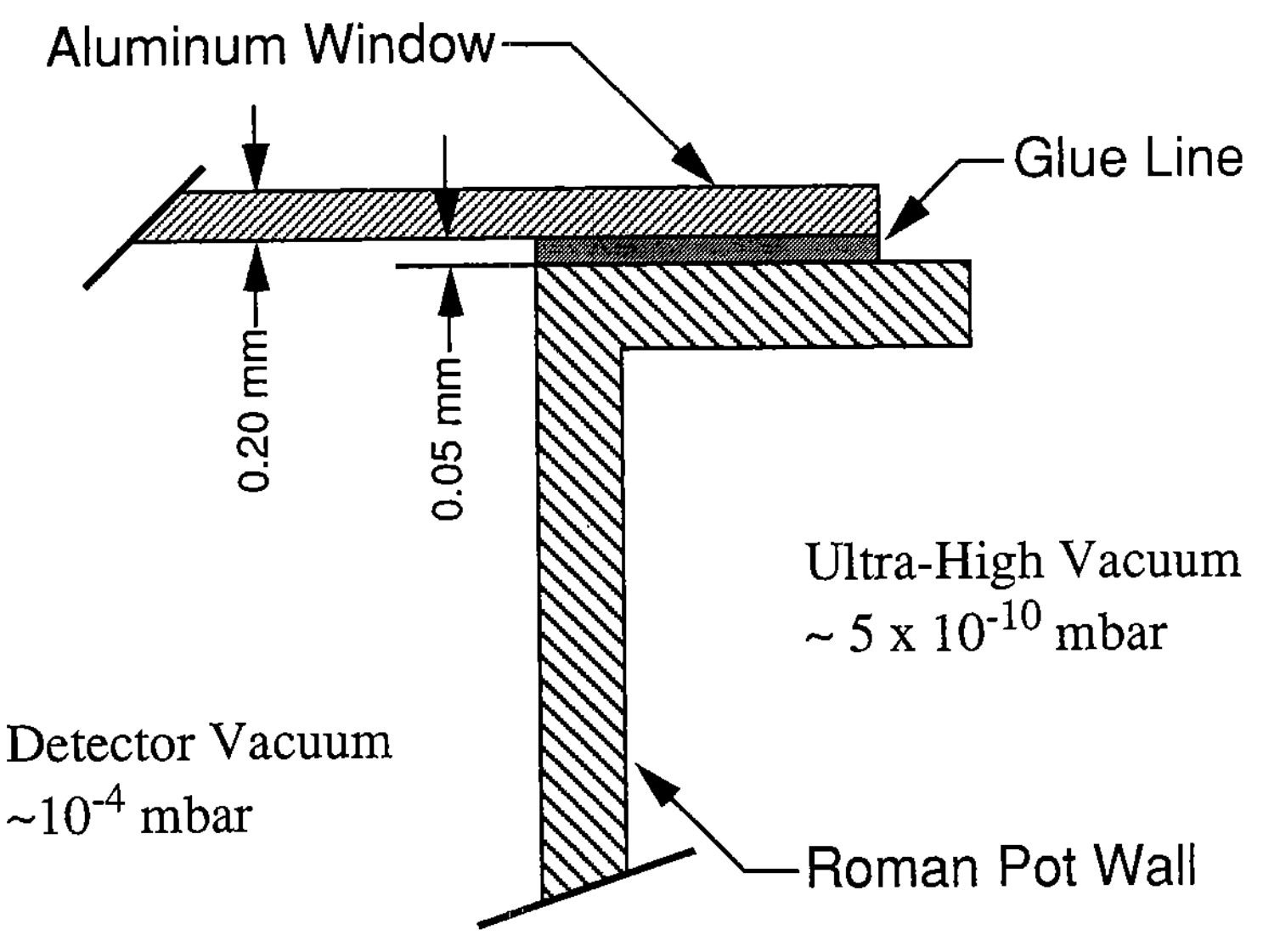

Figure 10: Detail of the joint between the $200 \mu \mathrm{m}$ window and the Roman Pot. The edge of the aluminum window, at the top of the sketch, is glued to a flange on the Roman Pot. Maintenance of the ultra-high vacuum in the SPS ring required the exposed glue surface be minimized. In this view, the detector volume is below the aluminum window and to the left of the Roman pot wall. 


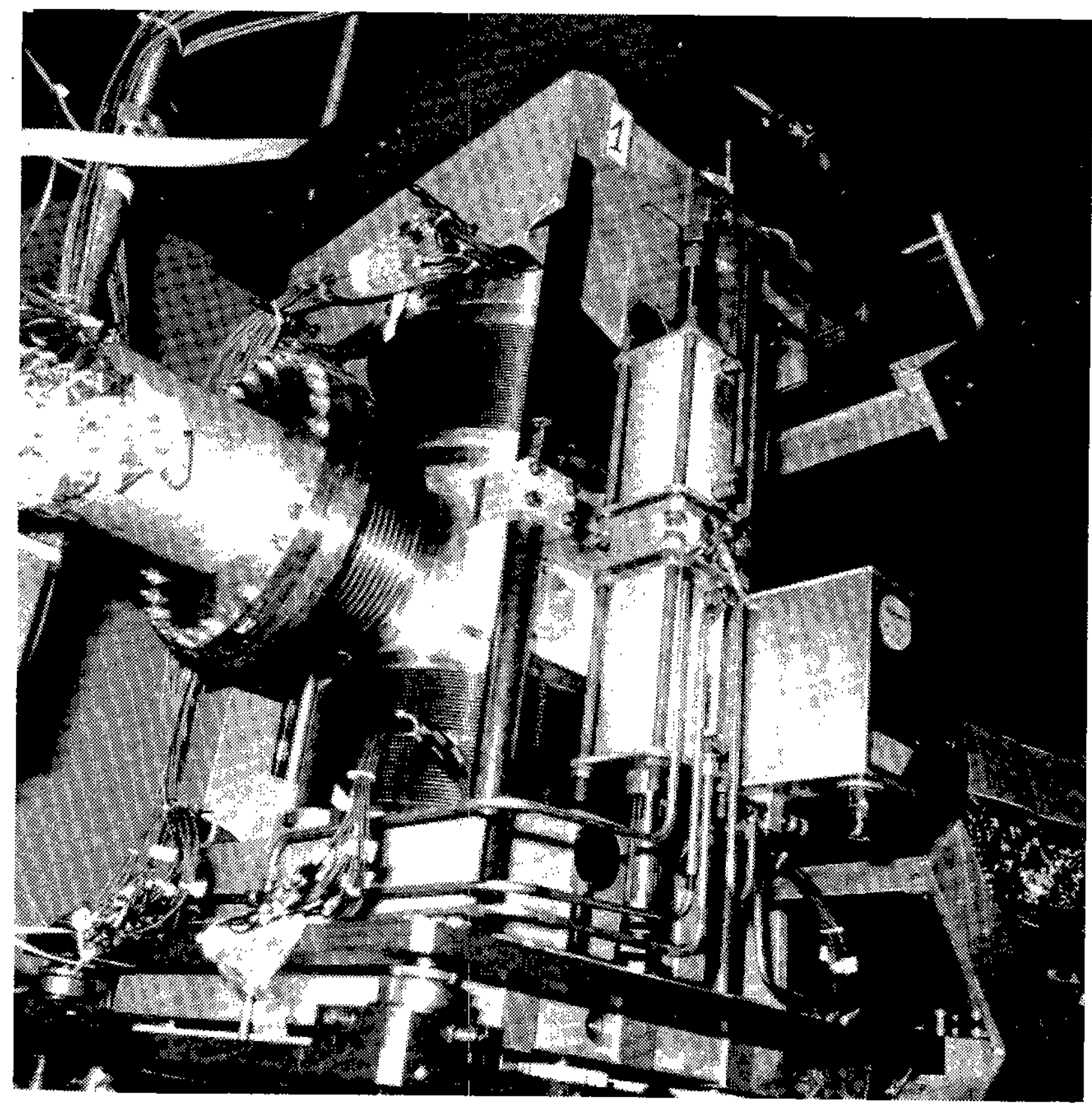

Figure 11: Pot positioning mechanism, after installation in the SPS. The beam pipe enters from the left of the photograph and attaches via a flange to the pot mechanism. The bellows, which permit vertical pot motion, are seen above and below this attachment. The cylinders mounted on the right side and tubes are part of a hydraulic system which compensates forces on the movable parts due to vacuum. 


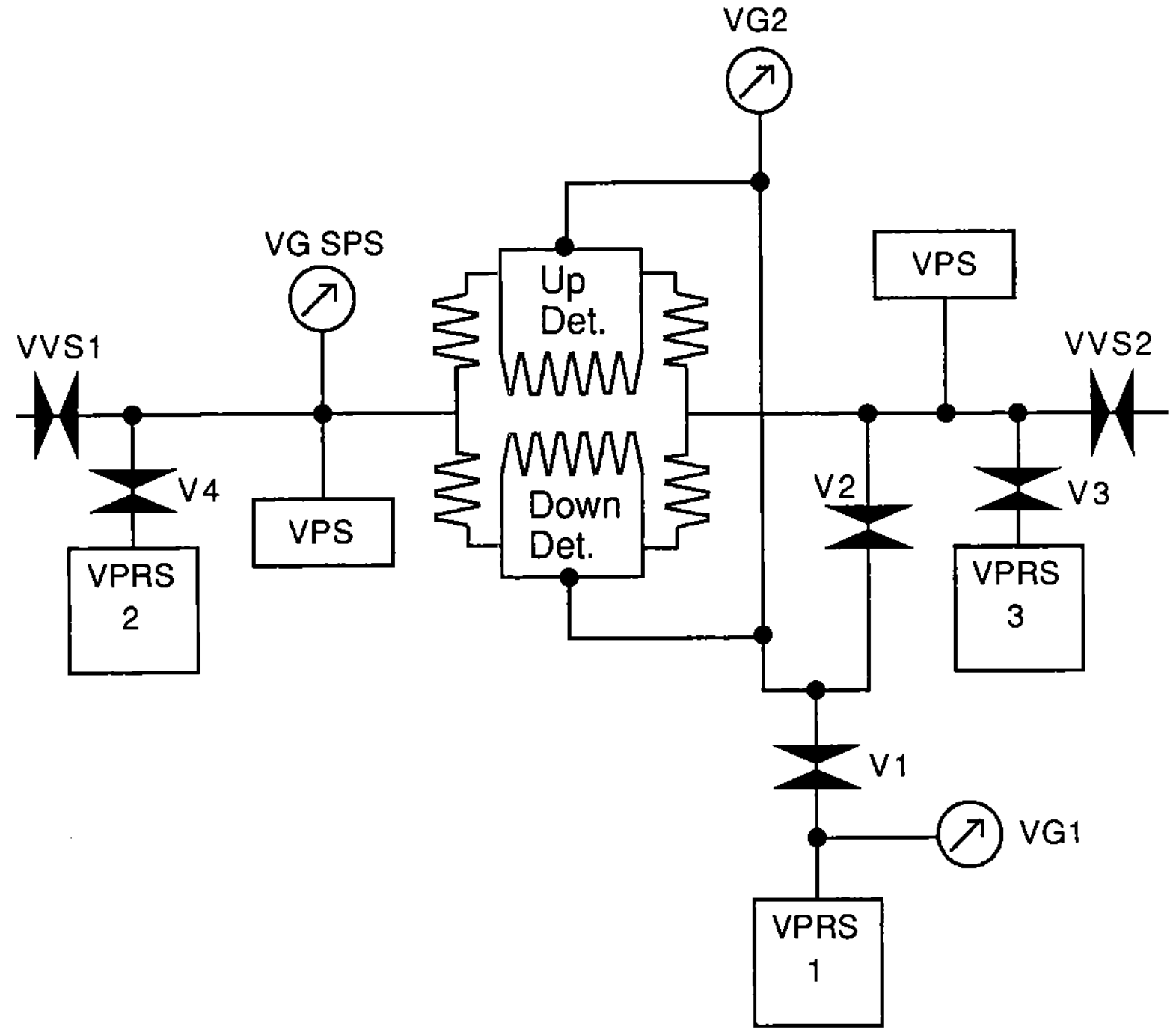

Figure 12: Schematic layout of the vacuum pumping system. The line between the SPS sector valves, VVS1 and VVS2, represents the SPS vacuum system with the Roman pot assembly in the center. VPRS1, VPRS2 and VPRS3 are turbomolecular pumps backed by rotary vane pumps. VG1, VG2 and VG-SPS are vacuum gauges and V1 to V4 are roughing valves. On each side of the pot assembly, a combination (VPS) of a $400 \ell / \mathrm{s}$ sputter-ion pump with a $1000 \ell / \mathrm{s}$ sublimation pump was installed for ultra high vacuum production. 


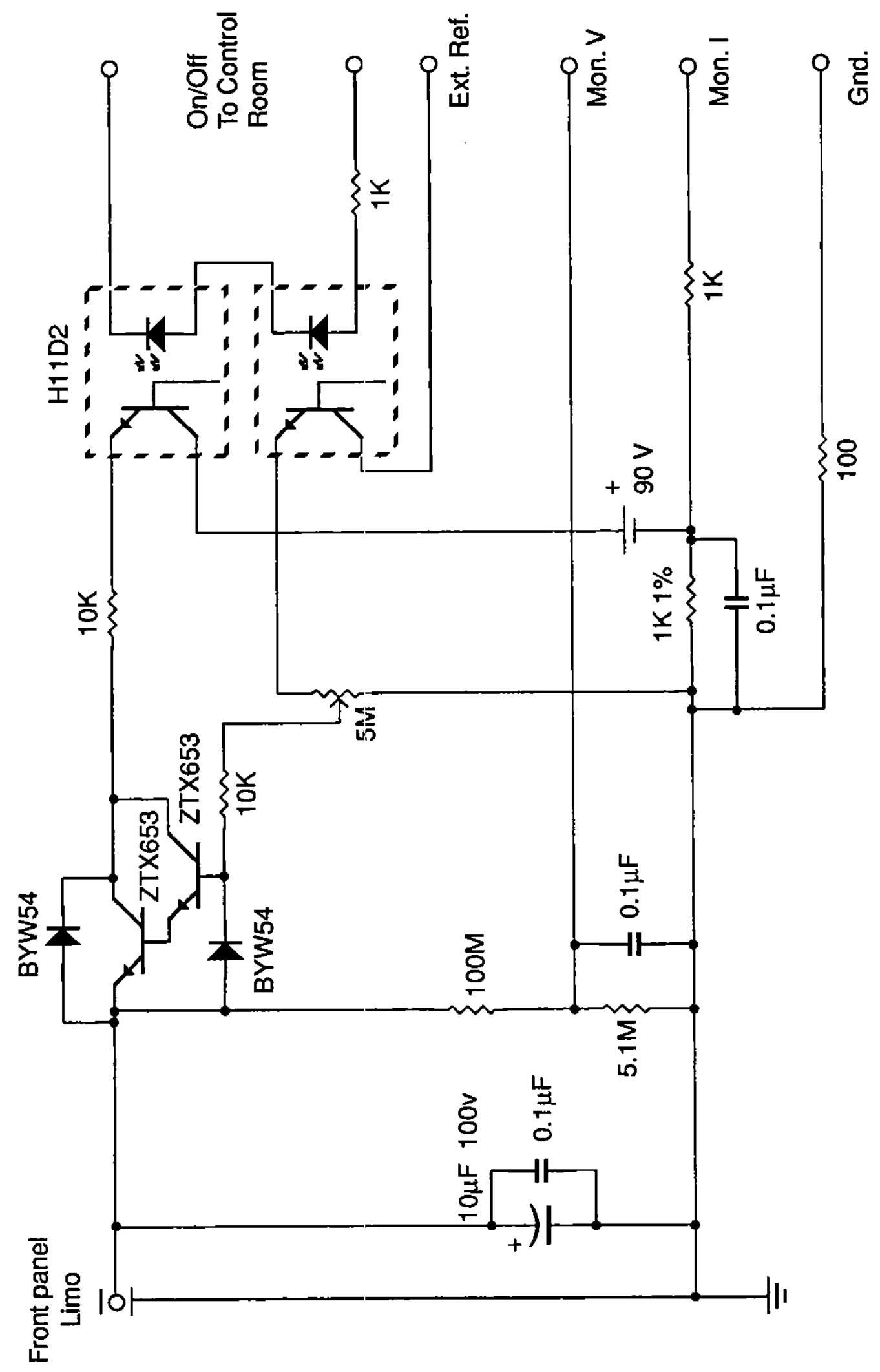

Figure 13: Power supply and control circuit. Detector bias was provided by $90 \mathrm{~V}$ batteries and controlled via a Darlington transistor pair. Remote control circuitry was isolated by optocouplers. 


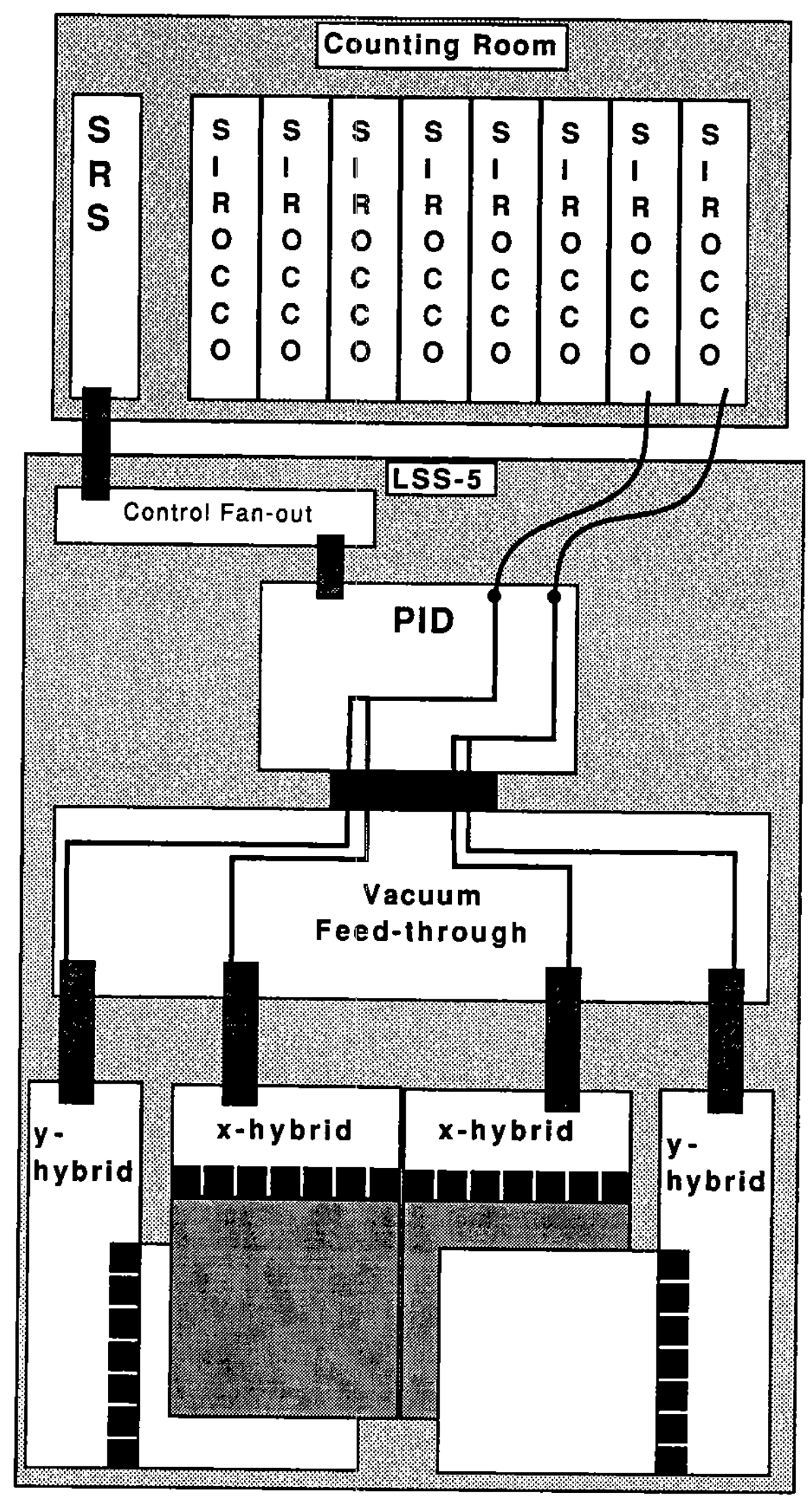

Figure 14: Schematic of readout and control paths for one detector half-plane. Control signals originate in the SRS, travel via an unshielded twisted-pair cable to the intersection region where they are fanned out to each of 12 PID boards. The PID boards relay the shaped signals to the readout hybrids through the vacuum bulkhead via a Feedthrough board and short lengths of Kapton cable. SVX analog output follows the same route from hybrids to PID boards, where $\mathrm{x}$-view and $\mathrm{y}$-view outputs are multiplexed, shaped, and sent to the counting room on shielded twisted-pair cables where digitization is accomplished by SIROCCO modules. 

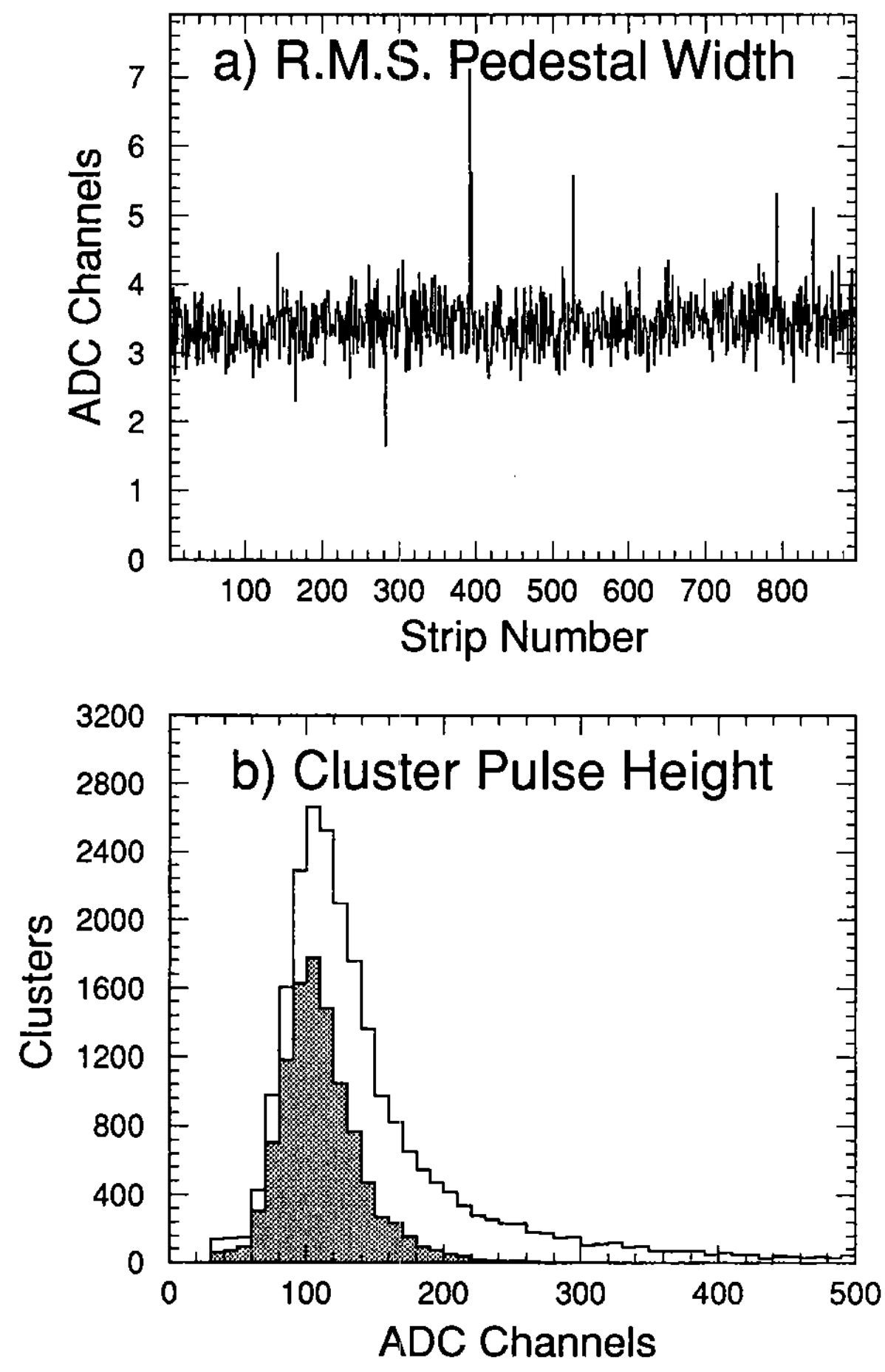

Figure 15: (a) The r.m.s. noise of individual channels of a typical detector vs. channel number. (b) Cluster pulse height distributions. Unshaded: clusters with 1, 2 or 3 strips; shaded: 2-strip clusters only. 

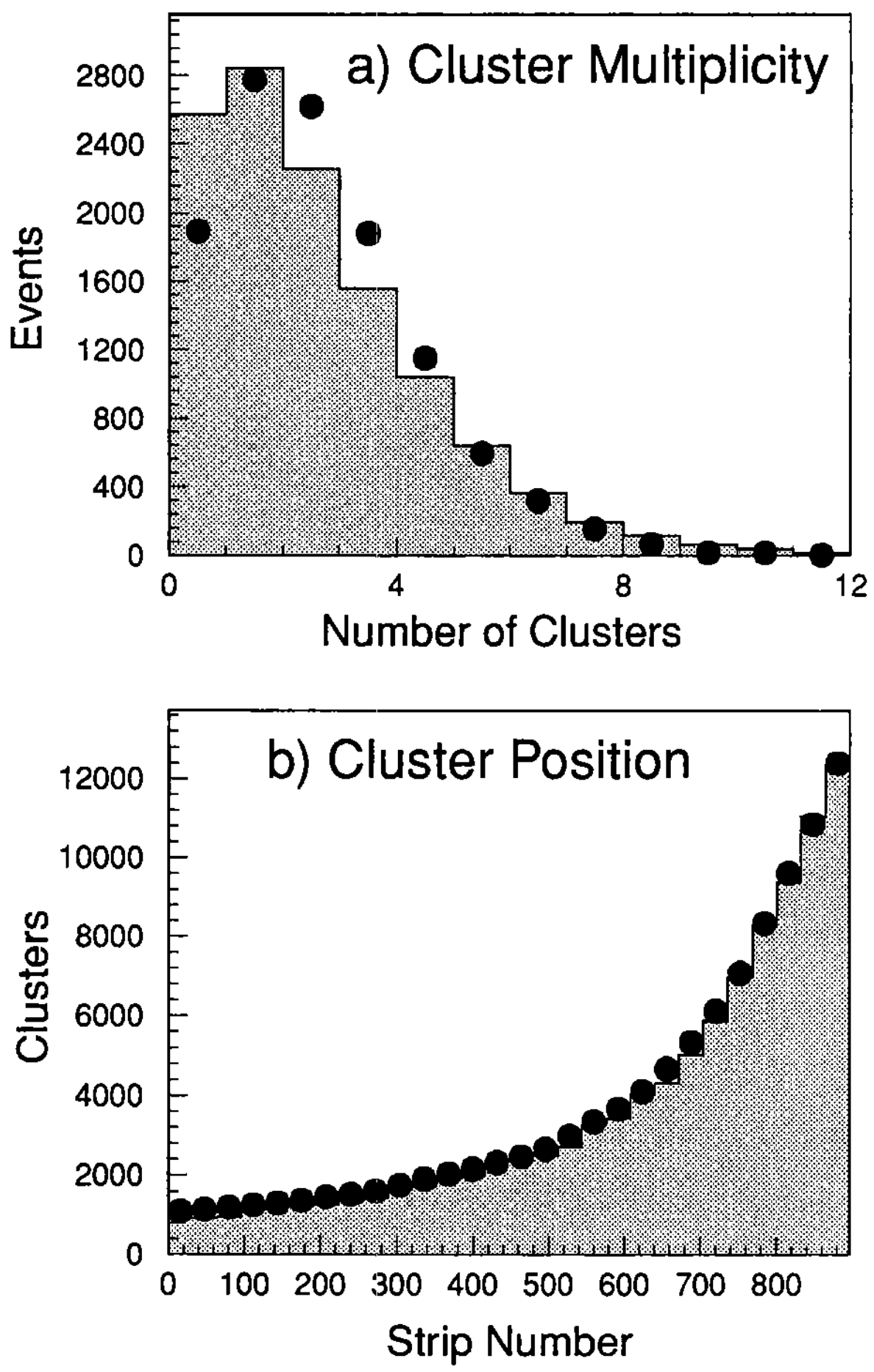

Figure 16: (a) Cluster multiplicity distribution on a typical detector. (b) Cluster position vs. channel number for the same detector. Channel 896 was closest to the beam. The shaded histograms are the data and the points are the results of the Monte Carlo simulation described in the text and normalized to the data. 


\section{Run 393 Event 891}

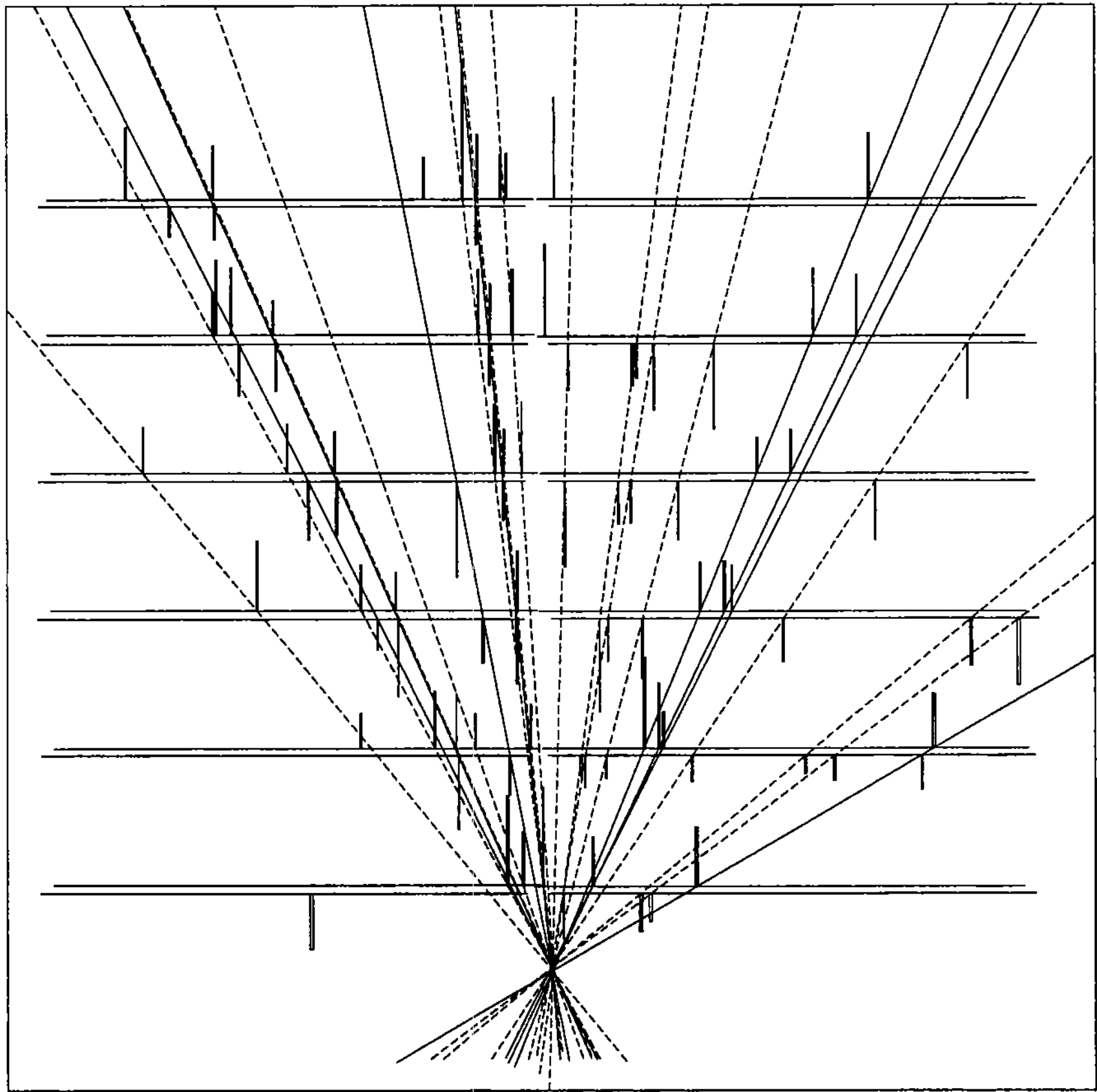

Figure 17: Display of the top view of a typical high-multiplicity event. Each silicon detector is represented by a horizontal line. All found clusters are shown as vertical lines, whose length is proportional to the cluster pulse height and whose width is proportional to the cluster width. The solid lines are tracks found in the upper half detector and dashed lines are those found in the lower half. Tracks are drawn to their z-intercepts and extended another $2 \mathrm{~cm}$. The distances between planes are $3.8 \mathrm{~cm}$, while the total detector width is twice $4.5 \mathrm{~cm}$. 
Run 393 Event $891 \quad$ Side View

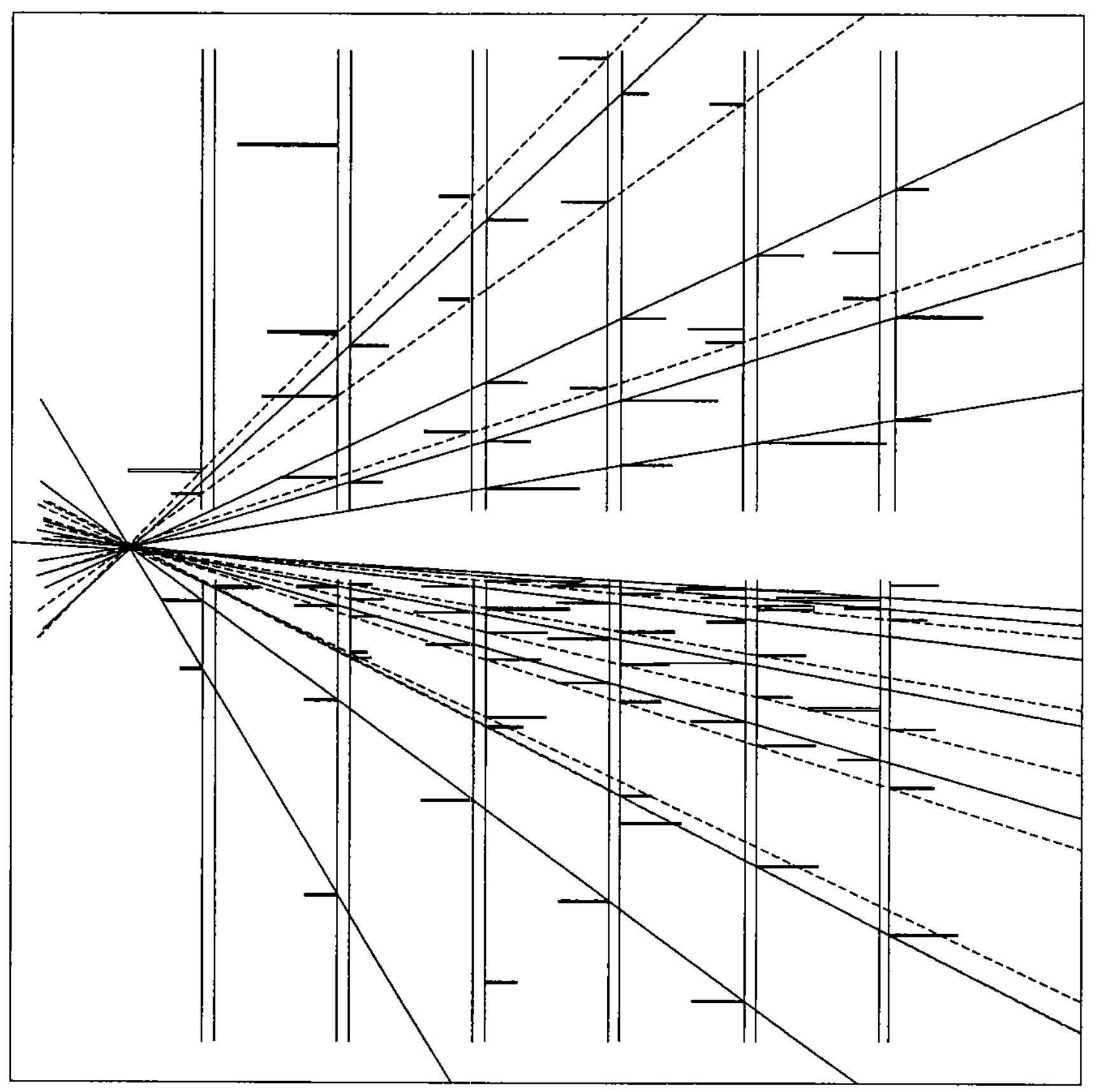

Figure 18: Side view of the event whose top view appears in Fig. 17. 


\section{Run 393 Event $9460 \quad$ Side View}

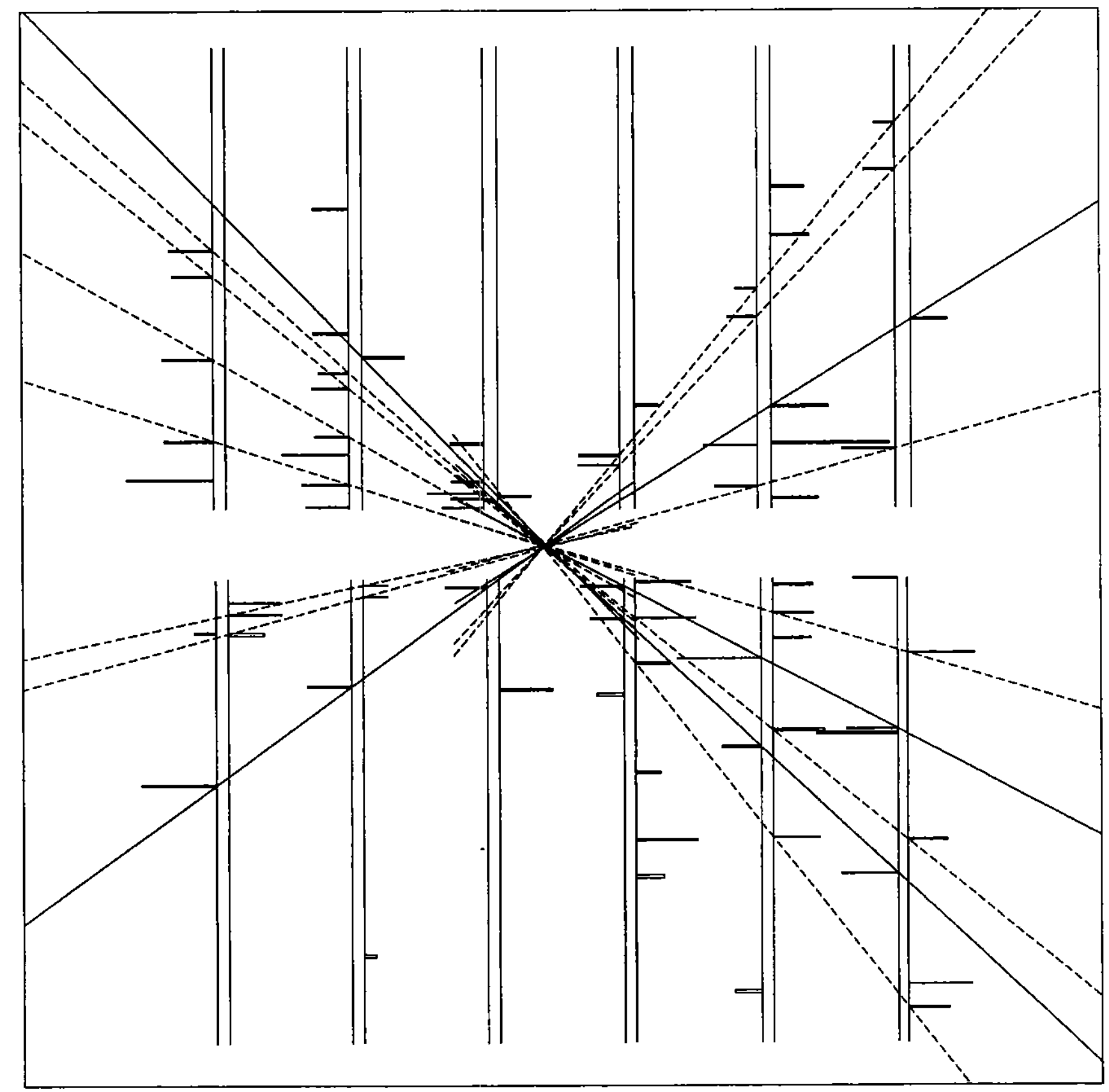

Figure 19: Side view of an event whose vertex occurs near the center of the detector. 


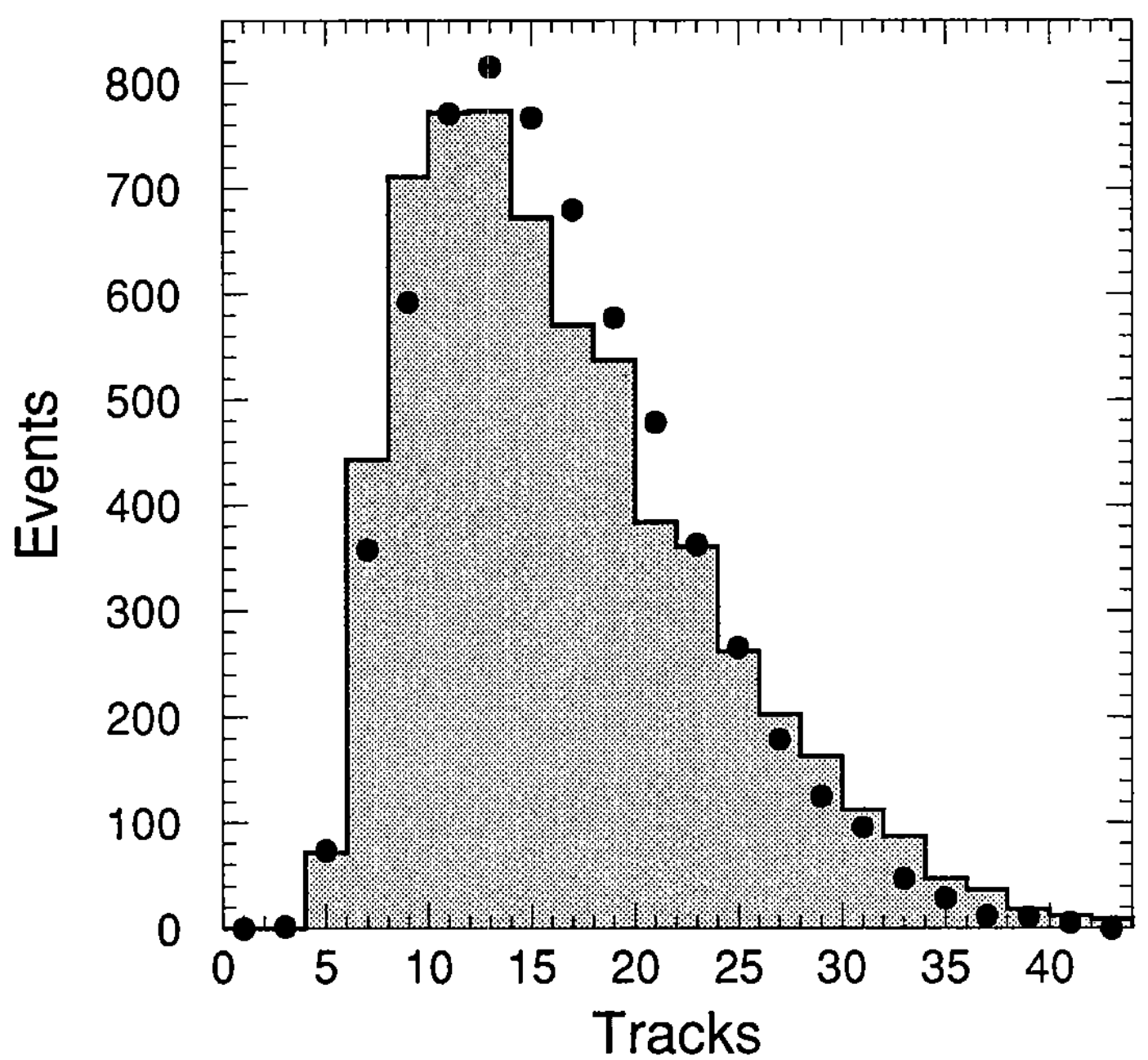

Figure 20: The distribution in total number of track $x-z$ and $y-z$ projections. The dots are from the Monte Carlo simulation described in the text. 


\section{Residual}
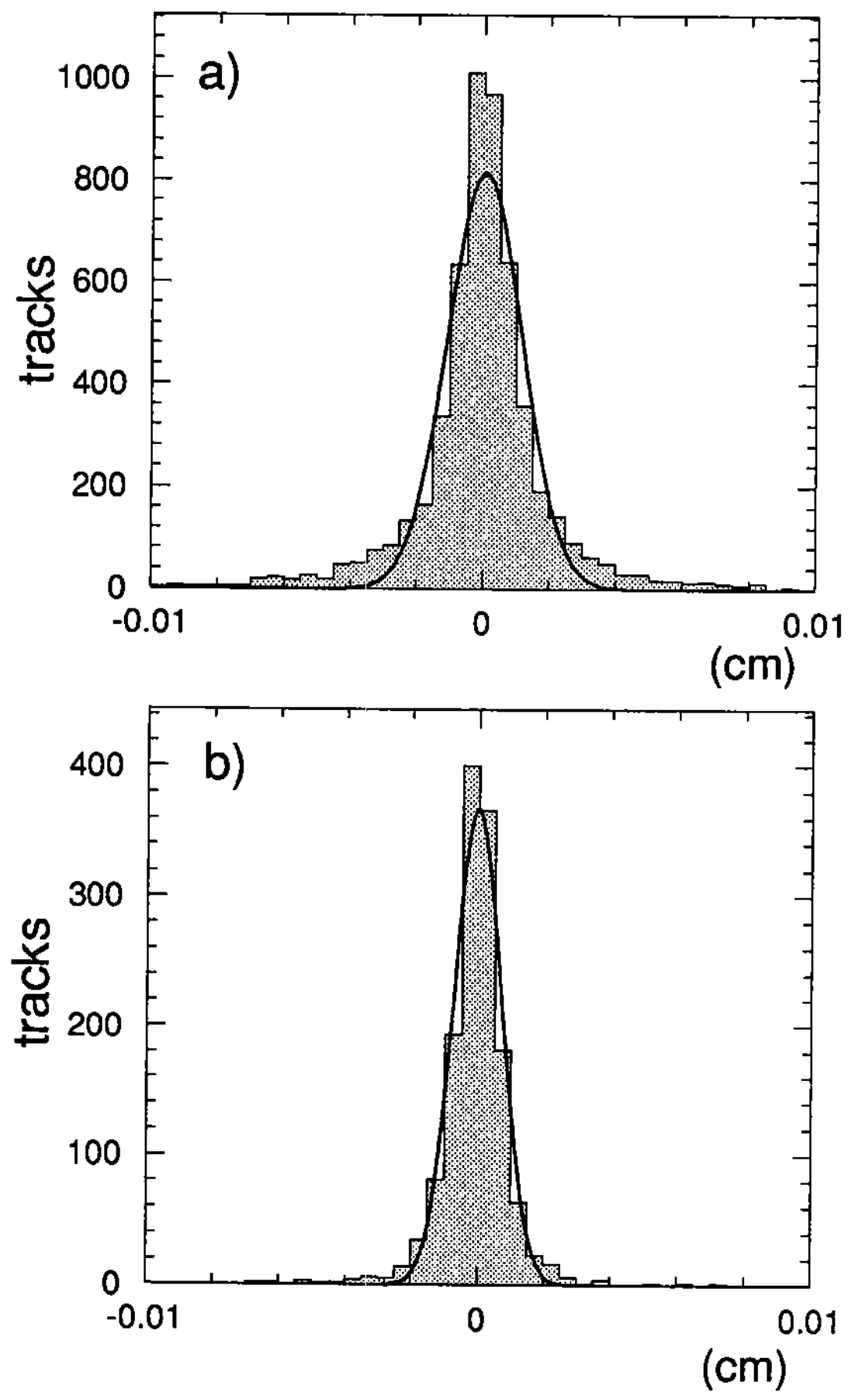

Figure 21: (a) Cluster residuals for one detector, using all found tracks. (b) Cluster residuals from the same detector, for tracks with slopes less than 0.1 and track $\chi^{2}$ per degree of freedom of less than 7. The curves are Gaussian fits with $\sigma=11.2$ and $7.2 \mu \mathrm{m}$, respectively. 


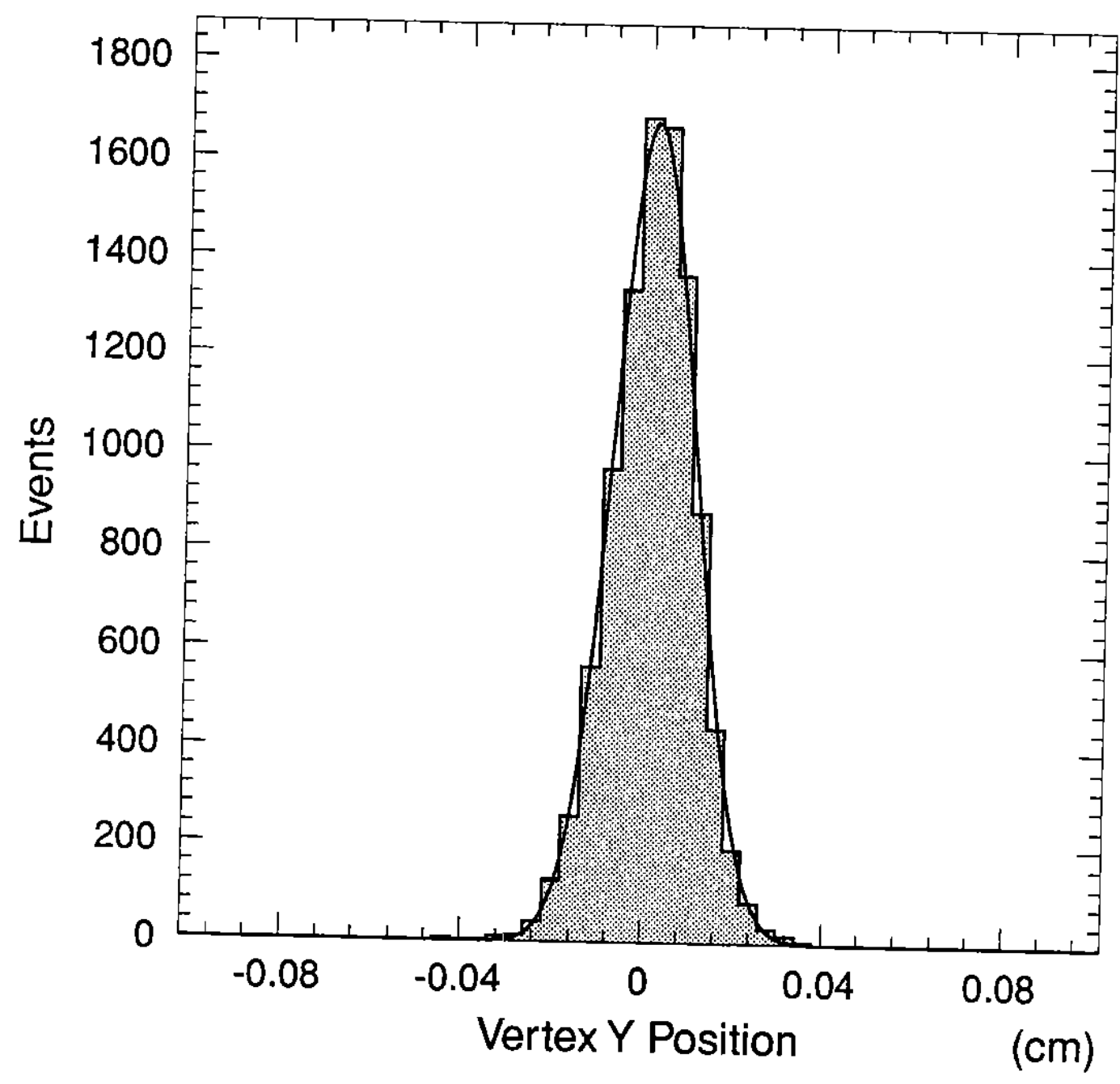

Figure 22: The y-projection of vertices found using all tracks in the event. The solid curve is a Gaussian fit with $\sigma=92 \mu \mathrm{m}$. 


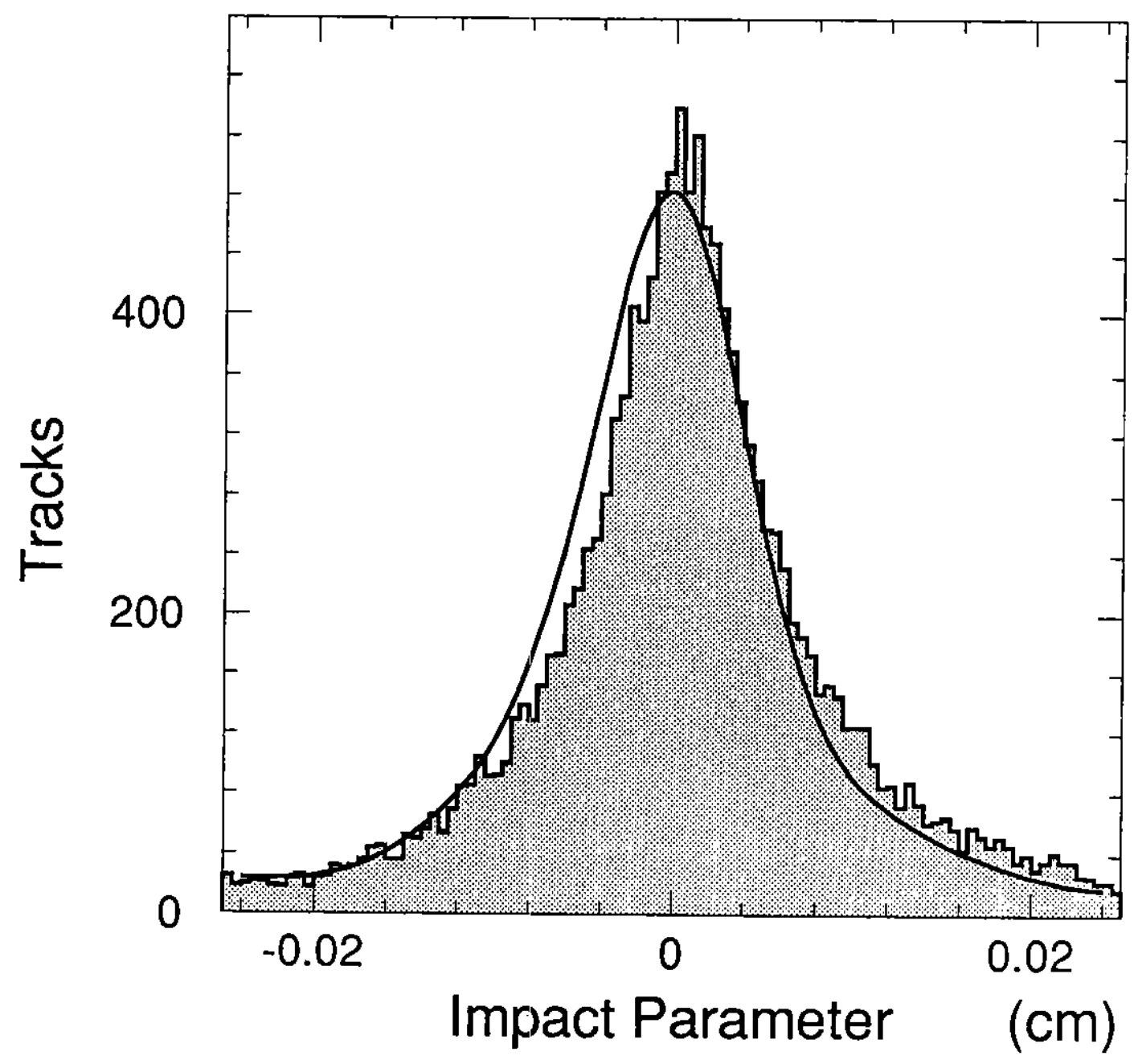

Figure 23: Impact parameter of tracks from a single quadrant. The impact parameter is defined as the difference between the fitted vertex $x$ (or $y$ ) coordinate and the track coordinate extrapolated to the $\mathrm{z}$ of the vertex. Tracks from all quadrants are used in the vertex fit. The non-Gaussian tail is due primarily to low momentum, badly measured or fake tracks. The FWHM is $90 \mu m$. The curve is the result of the Monte Carlo simulation described in the text. 


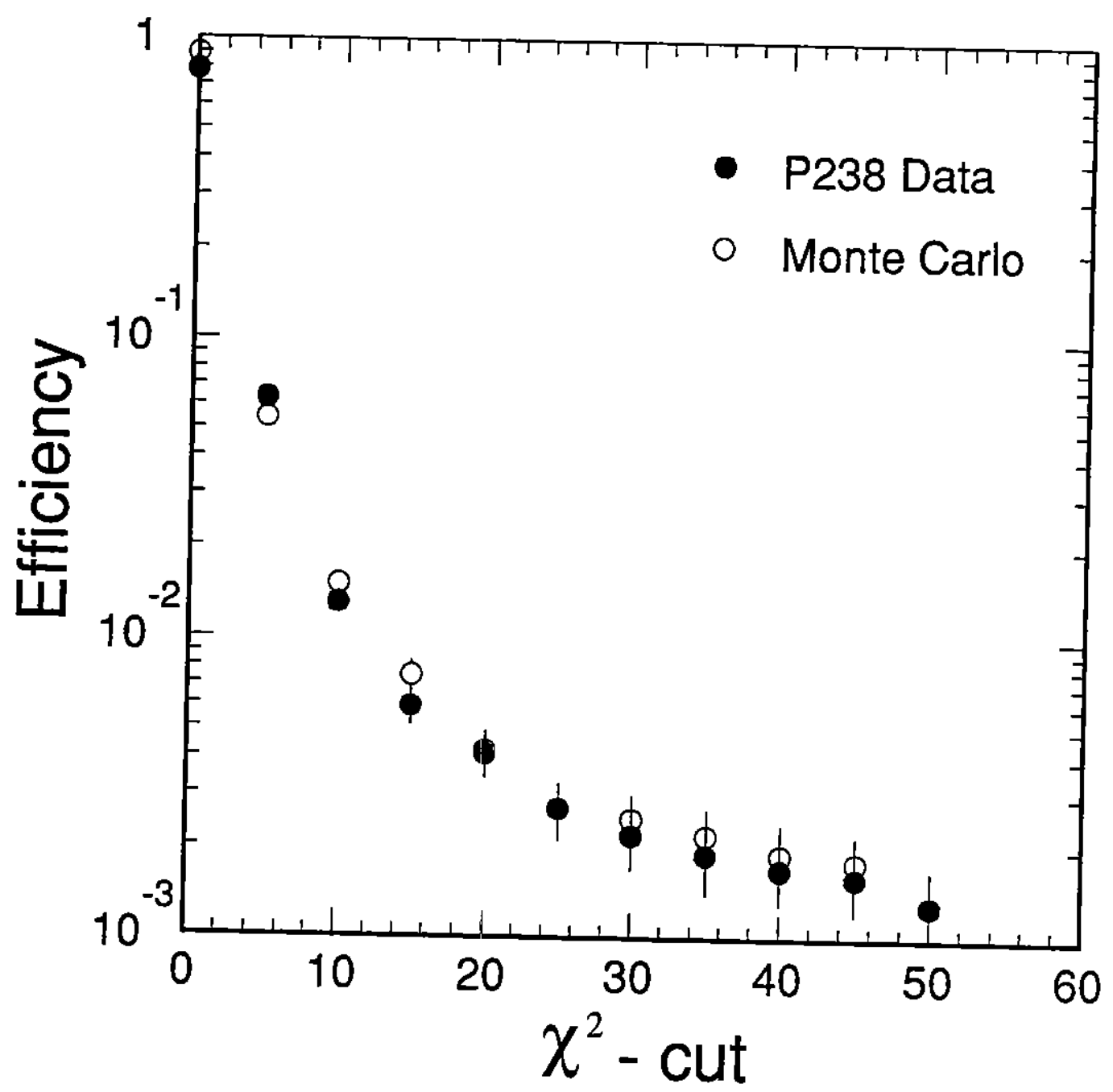

Figure 24: The efficiency for a P238-type trigger (see text) vs. $\chi^{2}$-cut.

Imprimé qu CERN. Prix: $\quad 1.15 \mathrm{Fr}$. 